\title{
29. BASALTS FROM THE EÄT PACIFIC RISE: AN EXAMPLE OF TYPICAL OCEANIC CRUST DEPLETED IN HYGROMAGMAPHILE ELEMENTS ${ }^{1}$
}

\author{
P. Cambon and H. Bougault, Centre National pour l'Exploitation des Océans, Centre Océanologique de Bretagne \\ B.P. 337-29273 Brest Cedex, France \\ J. L. Joron, Laboratoire P. Sue, Groupe des Sciences de la Terre-Cen Saclay \\ B.P. no. 2-91190 Gif-Sur-Yvette, France
}

M. Treuil, Université P. et M. Curie, Laboratoire de Géochimie Comparée et Systématique, 4, place Jussieu, Tour 24/25 E.3, 75230 Paris Cedex 05, France

\begin{abstract}
Samples of basalt collected on Leg 65 near $22^{\circ} \mathrm{N}$ on the East Pacific Rise all display the depleted light rare-earth pattern of "normal" oceanic crust. Consequently the $\mathrm{La} / \mathrm{Ta}$ ratio is close to 18 , as opposed to the value of 9 associated with the flat or enriched patterns found along parts of the Mid-Atlantic Ridge and the Emperor Seamount chain. The Leg 65 samples are chemically similar to those from the CYAMEX area at $21^{\circ} \mathrm{N}$ and to the Leg 54 samples from $9^{\circ} \mathrm{N}$, suggesting homogeneity of the upper mantle under the northern part of the East Pacific Rise over a minimum distance of about $1500 \mathrm{~km}$. The geochemistry of the rocks and their field relationships with respect to depth and distance from the axis of the Rise show no pattern of distribution linked to the degree of fractional crystallization and thus cast doubt on any possible model involving large, long-lived magma chambers at the axis of the Rise.
\end{abstract}

\section{INTRODUCTION}

The East Pacific Rise was drilled for the first time near $9^{\circ} \mathrm{N}$ during Deep Sea Drilling Project (DSDP) Leg 54. During Leg 65, several holes were drilled immediately south of the Tamayo Fracture Zone at about $22^{\circ} \mathrm{N}$, both on the eastern side of the Rise (Sites 482 and 485) and the western side (Site 483). Samples from close to the axis of the Rise were also obtained during the CYAMEX and RISE submersible expeditions in 1978 and 1979. In this paper, we present trace element data for the Leg 65 basalts-measured either by neutron activation analysis (NAA) or X-ray fluorescence spectrometry (XRF) in order to refine the definition of the basaltic units encountered in each hole. The geochemical properties of the basalts are also compared with data from Leg 54 and the CYAMEX expedition.

\section{RESULTS}

The major elements were analyzed on board the Glomar Challenger using the XRF unit from the Centre National pour l'Exploitation des Océans. In addition to the elements analyzed on earlier legs (e.g., Bougault, 1977; Melson et al., 1979), $\mathrm{Na}_{2} \mathrm{O}$ was analyzed on board for the first time on Leg 65. A new TlAP crystal was used which enhances the light-element counting rates compared to those obtained with an ADP crystal. With the TlAP crystal, the Mg counting time can be cut at the same time that the analytical accuracy is improved, and $\mathrm{Na}_{2} \mathrm{O}$ can be determined with an accuracy of $\pm 0.1 \%$. Three trace elements $(\mathrm{Ni}, \mathrm{Zr}$, and $\mathrm{Sr}$ ) were also measured on board (as on Legs 45, 46, and 55) to help dis-

\footnotetext{
${ }^{1}$ Lewis, B. T. R., Robinson, P., et al., Init. Repts. DSDP, 65: Washington (U.S. Govt. Printing Office).
}

criminate between different magmatic units. Of these, $\mathrm{Ni}$ has a high crystal/liquid partition coefficient; $\mathrm{Zr}$, which is a hygromagmaphile element, has a low partition coefficient; and $\mathrm{Sr}$, although also a hygromagmaphile element, is more or less sensitive to plagioclase crystallization (or melting).

Table 1 presents the major and trace element data available for the four rocks chosen as standards by the shipboard party. The $\mathrm{V}, \mathrm{Cr}, \mathrm{Co}, \mathrm{Zn}, \mathrm{Rb}, \mathrm{Y}$, and $\mathrm{Nb}$ concentrations were determined on shore using the X-ray fluorescence techniques described by Bougault et al. (1977); Sc, Co, Ni, Cs, La, Eu, Tb, Hf, Ta, Th, and U concentrations were measured using the neutron activation analysis procedures outlined by Treuil et al. (1973). The major element data for the rocks recovered on Leg 65 are presented in Table 2 and the trace element data in Table 3 . Tables 4 and 5 present the average trace element concentrations $(\bar{x})$ and standard deviations $(\sigma)$ for the homogeneous "chemical types" defined at Sites 482 and 483 , respectively. The tables showing trace element data include $\mathrm{Ti}, \mathrm{Mn}$, and $\mathrm{Fe}$ in order to have values shown in parts per million for all of the elements belonging to the first transition series presented together. The elements are arranged in order of increasing atomic number. The tables give $\mathrm{Co}$ and $\mathrm{Ni}$ concentrations from both XRF and NAA.

Data on trace elements confirm or refine the classification of the basaltic units identified on ship board. As far as possible, the "chemical types" defined by the shipboard party have been retained. Figures 1 and 2 show the distribution of these types versus depth for Sites 482 and 483 . The chemical types defined by trace element studies correlate well with those established at sea, although some differences are noted in the lower parts of Holes 482B and 483B. 
Table 1. Major and trace element composition of Leg 65 samples used as standards. $^{\mathrm{a}}$

\begin{tabular}{|c|c|c|c|c|}
\hline Components & $\begin{array}{l}482 \mathrm{C}-12-1 \\
92-118 \mathrm{~cm}\end{array}$ & $\begin{array}{l}483 \mathrm{~B}-8-3 \\
2-20 \mathrm{~cm}\end{array}$ & $\begin{array}{l}483 \mathrm{~B}-28-1 \\
42-59 \mathrm{~cm}\end{array}$ & $\begin{array}{l}485 \mathrm{~A}-25-1, \\
117-143 \mathrm{~cm}\end{array}$ \\
\hline \multicolumn{5}{|c|}{ Major elements (wt.\%) } \\
\hline $\mathrm{SiO}_{2}$ & 49.82 & 47.52 & 49.08 & 48.79 \\
\hline $\mathrm{Al}_{2} \mathrm{O}_{3}$ & 14.66 & 16.24 & 14.03 & 14.34 \\
\hline $\mathrm{Fe}_{2} \mathrm{O}_{3}$ & 11.20 & 9.69 & 12.24 & 12.80 \\
\hline $\mathrm{MnO}$ & 0.18 & 0.16 & 0.18 & 0.20 \\
\hline $\mathrm{MgO}$ & 7.81 & 9.19 & 7.65 & 7.07 \\
\hline $\mathrm{CaO}$ & 12.16 & 12.07 & 11.54 & 11.13 \\
\hline $\mathrm{Na}_{2} \mathrm{O}$ & 1.94 & 2.23 & 2.21 & 2.48 \\
\hline $\mathrm{K}_{2} \mathrm{O}$ & 0.07 & 0.04 & 0.10 & 0.08 \\
\hline $\mathrm{TiO}_{2}$ & 1.28 & 1.05 & 1.79 & 2.14 \\
\hline $\mathrm{P}_{2} \mathrm{O}_{5}$ & 0.15 & 0.12 & 0.19 & 0.21 \\
\hline Loss on ignition & & & & \\
\hline $\begin{array}{c}110^{\circ} \mathrm{C} \\
\text { Loss on ignition }\end{array}$ & 0.42 & 0.30 & 0.36 & 0.27 \\
\hline $1050^{\circ} \mathrm{C}$ & 0.42 & 1.38 & 0.64 & 0.35 \\
\hline Total & 100.11 & 99.99 & 100.01 & 99.86 \\
\hline \multicolumn{5}{|l|}{ Trace elements (ppm) } \\
\hline $\mathrm{v}$ & 313 & 227 & 350 & 387 \\
\hline $\mathrm{Cr}$ & 176 & 306 & 220 & 206 \\
\hline Co & 43 & 45 & 45 & 44 \\
\hline $\mathrm{Ni}$ & 55 & 96 & 166 & 59 \\
\hline $\mathrm{Rb}$ & $<1$ & $<1$ & $<1$ & $<1$ \\
\hline $\mathrm{Sr}$ & 92 & 135 & 108 & 103 \\
\hline $\mathrm{Y}$ & 36 & 29 & 46 & 56 \\
\hline $\mathrm{Zr}$ & 74 & 60 & 111 & 132 \\
\hline $\mathrm{Nb}$ & 1 & 1 & 3.5 & 3.5 \\
\hline
\end{tabular}

a Values determined by XRF analysis.

\section{Site 482}

Below Section 482B-18-2, for example, two different samples (482B-19-1, 96-100 cm and 482B-21-1, 62-64 $\mathrm{cm}$ ) stand out because of their high concentrations of hygromagmaphile elements. The samples are respectively classified as Chemical Types $\mathrm{C}$ and E. Other samples from this interval are classified as Chemical Type D. Among these samples, however, there are differences which lead to some elements having standard deviations which are higher than those reported in Tables 4 and 5 . The following subdivision of Chemical Type D is therefore proposed: Sample 482B-18-2, 99-101 cm has an Fe concentration of $82,000 \mathrm{ppm}$ compared with 71,000 ppm for the mean value for D and is thus assigned to subunit D' . Samples 482B-20-3, 24-26 cm and 482B-24$1,93-95 \mathrm{~cm}$ are assigned, in turn, to subunits $\mathrm{D}^{\prime \prime}$, and $\mathrm{D}^{\prime \prime \prime}$. Although these subdivisions may not be fundamental, they take into account differences (of $30 \mathrm{ppm}$ in $\mathrm{Sr}$ content between subunits $\mathrm{D}^{\prime \prime}$ and $\mathrm{D}^{\prime \prime \prime}$, for instance) that are significant from an analytical viewpoint.

It is interesting to note that Sample 482B-21-1, 62-64 cm (Type E) and Sample 482D-10-3, 14-16 cm are geochemically the same except for their $\mathrm{Cr}$ contents, but occur at widely separated levels ( $205 \mathrm{~m}$ and $155 \mathrm{~m}$, respectively). The only analyzed sample (Section 482F-4-3) from Hole $482 \mathrm{~F}$ corresponds to this chemical type.

\section{Site 483}

Two chemical types, Types $\mathrm{K}$ and $\mathrm{L}$, were defined by the shipboard party for grouping the basalts recovered from the lower part of Hole 483B, below Sample 483B$23-4,63-71 \mathrm{~cm}$. On the basis of the trace element data, however, all of the analyzed samples seem to be very similar: notable differences from Sections 483B-28-1 through $32-1$ and the remaining samples only concern $\mathrm{Cr}, \mathrm{Ni}$, and the hygromagmaphile elements. We thus prefer to assign all of the samples to only one chemical type and to subdivide the subunits into $K$ and $\mathrm{K}^{\prime}$. We include in subunit $\mathrm{K}$ Sections 483B-23-4 through 27-3 and Section 483B-32-3, and assign to $\mathrm{K}^{\prime}$ Sections 483B-28-1 through 32-1. The observed differences suggest that subunit $\mathrm{K}$ was derived from $\mathrm{K}^{\prime}$ by fractional crystallization or that $\mathrm{K}^{\prime}$ contains more ferromagnesian minerals than subunit $\mathrm{K}$.

Similarly, in Hole 483, Chemical Type F defined on board ship incorporates the sequence from Section 20-1 through Section 23-2. We suggest that this unit should be divided into subunits $\mathrm{F}$ (Sections 483-20-1 through 21-2) and $\mathrm{F}^{\prime}$ (Sections 483-22-1 to 23-2). The comments made concerning subunits $\mathrm{K}$ and $\mathrm{K}^{\prime}$ in Hole 483B also apply to the subdivisions $\mathrm{F}$ and $\mathrm{F}^{\prime}$ in Hole 483 . Section 25-1 in Hole 483 (near the $198 \mathrm{~m}$ level) belongs to Chemical Type A, which occurs at about the 110-m level in Holes 483 and 483B.

\section{Site $\mathbf{4 8 5}$}

Unlike the samples from Sites 482 and 483 , the basalts from Hole 485A have not been grouped into chemical units. Except for the obvious difference between the uppermost sample (Section 485A-11-3) and the basalts obtained deeper in the section, the differences between samples are not clear enough to justify classification into different chemical types. The variable Ni concentrations observed could be the result of variations in olivine content.

\section{TRACE ELEMENT GEOCHEMISTRY, FIELD DATA, AND SIZE OF MAGMA CHAMBERS}

In a recent discussion on the East Pacific Rise at $22^{\circ} \mathrm{N}$ (RISE Project Group, 1980), it was noted that there is no correlation between the degree of fractionation of lavas and their age or distance from the Rise axis. From the present study, it is also evident that there is no clear relationship between fractionation and the vertical distribution of chemical units in the crust, at least in the holes drilled on Leg 65 . In nearly all of the samples examined, the $\mathrm{Ni}$ concentration varies between 50 and $100 \mathrm{ppm}$. This range of variation can be attributed, in part, to variations in olivine content, but it also requires the removal of olivine by crystallization processes. For similar $\mathrm{Ni}$ concentrations, the $\mathrm{Cr}$ content varies between $190 \mathrm{ppm}$ and $380 \mathrm{ppm}$. Once again, variations in the abundance of olivine, spinel, and clinopyroxene phenocrysts cannot entirely explain the observed values, and the removal of varying proportions of ferromagnesian minerals during fractional crystallization must also be invoked.

In addition, it was noted by the shipboard party that the uppermost unit of basalt in Hole 485A, represented by Sample $485 \mathrm{~A}-11-3,82-83 \mathrm{~cm}$, is close in composition to a primitive liquid. This observation is confirmed by 
Table 2. Major element composition of basalts, Leg $65 .^{\mathrm{a}}$

\begin{tabular}{|c|c|c|c|c|c|c|c|c|c|c|c|c|}
\hline $\begin{array}{c}\text { Sample } \\
\text { (interval in } \mathrm{cm} \text { ) }\end{array}$ & $\mathrm{SiO}_{2}$ & $\mathrm{TiO}_{2}$ & $\mathrm{Al}_{2} \mathrm{O}_{3}$ & $\mathrm{Fe}_{2} \mathrm{O}_{3}{ }^{\mathrm{b}}$ & $\mathrm{MnO}$ & $\mathrm{MgO}$ & $\mathrm{CaO}$ & $\mathrm{Na}_{2} \mathrm{O}$ & $\mathrm{K}_{2} \mathrm{O}$ & $\mathrm{P}_{2} \mathrm{O}_{5}$ & $\mathrm{LOI}^{\mathrm{c}}$ & Total \\
\hline Hole 482B & & & & & & & & & $\because$ & & & \\
\hline $10-7,14-16$ & 43.44 & 1.77 & 13.96 & 13,05 & 0.23 & 6.68 & 9.57 & 2.58 & 0.08 & 0.17 & 7.78 & 99.34 \\
\hline $11-1,23-25$ & 48.98 & 1.76 & 13.93 & 11.39 & 0.20 & 7.48 & 11.96 & 2.36 & 0.08 & 0.17 & 1.61 & 99.92 \\
\hline $12-2,135-137$ & 49.27 & 1.74 & 14.04 & 11.61 & 0.18 & 7.91 & 11.64 & 2.18 & 0.08 & 0.18 & 1.11 & 99.94 \\
\hline $14-2,121-124$ & 48.86 & 1.39 & 14.42 & 11.07 & 0.18 & 7.80 & 12.01 & 2.19 & 0.05 & 0.13 & 1.47 & 99.57 \\
\hline $15-1,135-139$ & 48.85 & 1.30 & 14.48 & 10.99 & 0.18 & 7.95 & 11.92 & 2.00 & 0.04 & 0.12 & 1.73 & 99.56 \\
\hline $15-3,117-119$ & 49.30 & 1.26 & 14.29 & 10.95 & 0.16 & 7.80 & $12.16^{\prime}$ & 2.17 & 0.05 & 0.11 & 1.33 & 99.58 \\
\hline $16-1,36-38$ & 49.44 & 1.27 & 14.44 & 10.94 & 0.16 & 7.80 & 12.10 & 2.08 & 0.05 & 0.11 & 1.12 & 99.51 \\
\hline $17-2,111-113$ & 49.36 & 1.30 & 14.27 & 11.35 & 0.19 & 7.73 & 12.36 & 1.98 & 0.02 & 0.12 & 1.04 & 99.72 \\
\hline $18-1,18-20$ & 49.69 & 1.30 & 14.06 & 11.26 & 0.17 & 7.88 & 11.94 & 2.18 & 0.06 & 0.11 & 1.12 & 99.77 \\
\hline $18-2,99-101$ & 48.91 & 1.54 & 14.10 & 11.81 & 0.19 & 7.55 & 12.05 & 2.03 & 0.04 & 0.13 & 1.10 & 99.45 \\
\hline $19-1,96-100$ & 47.99 & 2.20 & 16.09 & 11.56 & 0.12 & 7.14 & 7.12 & 3.04 & 0.06 & 0.21 & 3.77 & 99.30 \\
\hline $20-3,24-26$ & 48.55 & 1.54 & 15.33 & 9.97 & 0.16 & 7.31 & 11.38 & 2.39 & 0.04 & 0.14 & 2.58 & 99.39 \\
\hline $21-1,62-64$ & 48.22 & 1.88 & 13.84 & 11.70 & 0.16 & 7.47 & 11.20 & 2.43 & 0.06 & 0.16 & 1.82 & 98.94 \\
\hline $21-3,74-76$ & 49.00 & 1.61 & 15.16 & 10.65 & 0.17 & 7.15 & 12.03 & 2.37 & 0.03 & 0.16 & 1.40 & 99.73 \\
\hline $22-2,34-36$ & 49.27 & 1.56 & 15.57 & 10.64 & 0.16 & 6.94 & 12.04 & 2.36 & 0.03 & 0.15 & 1.47 & 100.19 \\
\hline $22-4,34-36$ & 48.61 & 1.51 & 14.87 & 10.37 & 0.16 & 6.68 & 11.88 & 2.24 & 0.06 & 0.14 & 2.79 & 99.31 \\
\hline $24-1,93-95$ & 48.10 & 1.63 & 15.42 & 10.22 & 0.15 & 7.20 & 10.35 & 2.46 & 0.05 & 0.14 & 3.62 & 99.34 \\
\hline Hole $482 \mathrm{C}$ & & & & & & & & & & & & \\
\hline $9-1,121-123$ & 48.71 & 1.77 & 14.25 & 11.58 & 0.20 & 7.57 & 11.63 & 2.06 & 0.08 & 0.17 & 1.96 & 99.98 \\
\hline $10-2,73-75$ & 48.83 & 1.71 & 14.28 & 11.41 & 0.19 & 7.94 & 11.45 & 2.06 & 0.06 & 0.16 & 1.81 & 99.90 \\
\hline $11-1,138-139$ & 48.78 & 1.73 & 14.34 & 11.08 & 0.17 & 7.82 & 11.82 & 2,27 & 0.07 & 0.17 & 1.46 & 99.71 \\
\hline $11-1,115-117$ & 48.45 & 1.78 & 14.42 & 11.36 & 0.17 & 7.68 & 11.67 & 2.17 & 0.06 & 0.17 & 1.56 & 99.49 \\
\hline $11-2,16-18$ & 47.76 & 1.32 & 13.45 & 10.58 & 0.15 & 9.57 & 9.21 & 2.53 & 0.07 & 0.14 & 4.66 & 99.44 \\
\hline $11-2,21-24$ & 47.32 & 1.19 & 13.74 & 11.51 & 0.13 & 10.25 & 8.31 & 2.40 & 0.08 & 0.12 & 4.15 & 99.20 \\
\hline $11-3,141-143$ & 49.17 & 1.79 & 13.85 & 11.66 & 0.18 & 7.75 & 11.62 & 2.22 & 0.07 & 0.17 & 1.46 & 99.94 \\
\hline $12-1,11-13$ & 49.22 & 1.30 & 14.35 & 11.20 & 0.18 & 8.03 & 11.88 & 1.85 & 0.05 & 0.12 & 1.04 & 99.22 \\
\hline $12-2,95-97$ & 49.49 & 1.30 & 14.41 & 11.33 & 0.18 & 7.81 & 12.15 & 1.98 & 0.03 & 0.11 & 1.19 & 99.98 \\
\hline $13-1,22-24$ & 48.70 & 1.30 & 14.56 & 10.47 & 0.15 & 8.26 & 11.43 & 2.05 & 0.03 & 0.10 & 2.28 & 99.33 \\
\hline $13-1,125-127$ & 49.23 & 1.29 & 14.21 & 11.13 & 0.17 & 7.79 & 12.05 & 2.02 & 0.04 & 0.11 & 1.33 & 99.37 \\
\hline $13-3,108-110$ & 49.23 & 1.30 & 14.30 & 11.27 & 0.18 & 7.66 & 12.10 & 2.03 & 0.05 & 0.12 & 1.09 & 99.33 \\
\hline $14-1,40-42$ & 49.40 & 1.28 & 14.27 & 11.06 & 0.17 & 7.67 & 12.16 & 1.97 & 0.04 & 0.12 & 1.31 & 99.45 \\
\hline $14-4,127-129$ & 49.25 & 1.29 & 14.19 & 11.22 & 0.17 & 7.93 & 12.14 & 1.93 & 0.05 & 0.12 & 1.10 & 99.39 \\
\hline $15-1,44-46$ & 49.41 & 1.29 & 14.20 & 11.19 & 0.18 & 7.74 & 12.09 & 2.05 & 0.04 & 0.11 & 1.11 & 99.41 \\
\hline $15-2,132-134$ & 49.21 & 1.27 & 14.14 & 11.03 & 0.17 & 7.88 & 12.05 & 1.95 & 0.05 & 0.12 & 1.53 & 99.40 \\
\hline $15-3,97-99$ & 49.52 & 1.27 & 14.35 & 11.16 & 0.18 & 7.81 & 12.12 & 1.95 & 0.02 & 0.12 & 1.18 & 99.68 \\
\hline $15-4,119-121$ & 49.61 & 1.26 & 14.28 & 11.10 & 0.18 & 7.68 & 12.16 & 2.00 & 0.05 & 0.11 & 1.18 & 99.61 \\
\hline Hole 482D & & & & & & & & & t & & & \\
\hline $8-1,30$ & 46.24 & 1.79 & 14.35 & 10.90 & 0.25 & 6.40 & 10.56 & 2.08 & 0.46 & 0.17 & 5.61 & 98.81 \\
\hline $8-1,109-111$ & 48.93 & 1.75 & 14.04 & 11.51 & 0.19 & 7.57 & 11.84 & 2.06 & 0.04 & 0.16 & 1.81 & 99.90 \\
\hline $9-1,48-50$ & 49.21 & 1.78 & 13.86 & 11.59 & 0.20 & 7.70 & 11.80 & 2.17 & 0.03 & 0.17 & 1.46 & 99.97 \\
\hline $9-3,50-52$ & 48.93 & 1.80 & 13.90 & 11.84 & 0.20 & 7.44 & 11.73 & 2.39 & 0.05 & 0.17 & 1.31 & 99.76 \\
\hline $10-2,52-55$ & 49.31 & 1.76 & 13.84 & 11.60 & 0.19 & 7.64 & 11.78 & 2.42 & 0.05 & 0.17 & 1.39 & 100.15 \\
\hline $10-2,109-112$ & 49.10 & 1.77 & 13.95 & 11.40 & 0.18 & 7.72 & 11.54 & 2.35 & 0.07 & 0.16 & 1.88 & 100.12 \\
\hline $10-3,14-16$ & 49.36 & 1.90 & 13.89 & 11.92 & 0.19 & 7.51 & 11.33 & 2.65 & 0.04 & 0.17 & 1.32 & 100.27 \\
\hline $10-3,101-103$ & 48.49 & 1.41 & 14.80 & 11.05 & 0.18 & 7.29 & 11.99 & 2.52 & 0.05 & 0.13 & 2.16 & 100.07 \\
\hline $11-1,135-137$ & 48.65 & 1.41 & 14.32 & 11.10 & 0.18 & 7.86 & 11.91 & 2.35 & 0.04 & 0.12 & 1.73 & 99.67 \\
\hline $12-1,105-107$ & 48.88 & 1.39 & 14.44 & 11.17 & 0.18 & 7.90 & 11.84 & 2.24 & 0.04 & 0.13 & 1.11 & 99.32 \\
\hline $12-2,50-52$ & 49.09 & 1.29 & 14.15 & 11.24 & 0.18 & 8.09 & 12.10 & 2.26 & 0.03 & 0.11 & 1.25 & 99.79 \\
\hline $12-2,82-84$ & 49.31 & 1.29 & 14.17 & 11.17 & 0.17 & 8.02 & 12.00 & 2.17 & 0.03 & 0.11 & 1.11 & 99.55 \\
\hline $12-3,118-120$ & 49.35 & 1.27 & 14.43 & 11.06 & 0.17 & 7.64 & 12.18 & 2.24 & 0.03 & 0.11 & 1.04 & 99.52 \\
\hline $13-1,124-127$ & 49.02 & 1.34 & 14.73 & 10.51 & 0.16 & 8.24 & 11.42 & 2.41 & 0.03 & 0.12 & 1.80 & 99.78 \\
\hline $13-3,4-6$ & 49.07 & 1.31 & 14.13 & 11.16 & 0.17 & 8.67 & 11.57 & 2.36 & 0.06 & 0.11 & 1.60 & 100.2 \\
\hline Hole $482 \mathrm{~F}$ & & & & & & & & & & & & \\
\hline $4-3,131-137$ & 49.05 & 1.95 & 13.75 & 12.28 & 0.19 & 7.52 & 11.31 & 2.41 & 0.08 & 0.19 & 1.11 & 99.84 \\
\hline Hole 483 & & & & & & & & & & & & \\
\hline $13-4,17-19$ & 49.37 & 1.27 & 15. & 10.8 & 0.16 & 7.18 & 12.2 & 2.22 & 0.05 & 0.1 & 1.04 & 100.34 \\
\hline $14-1,35-37$ & 48.25 & 1.18 & 14.48 & 10.54 & 0.23 & 8.23 & 12.23 & 2.38 & 0.07 & 0.11 & 2.08 & 99.78 \\
\hline $14-2,12-14$ & 49.06 & 1.20 & 14.63 & 10.42 & 0.16 & 7.88 & 12.25 & 2.55 & 0.06 & 0.11 & 1.53 & 99.85 \\
\hline $15-1,21-24$ & 49.01 & 1.19 & 14.59 & 10.49 & 0.17 & 8.43 & 12.44 & 2.44 & 0.05 & 0.11 & 1.04 & 99.96 \\
\hline $15-2,96-98$ & 48.62 & 1.19 & 14.58 & 10.29 & 0.16 & 8.73 & 12.50 & 2.48 & 0.05 & 0.11 & 1.18 & 99.89 \\
\hline $15-3,7-9$ & 49.75 & 1.37 & 14.38 & 10.87 & 0.17 & 7.59 & 12.09 & 2.47 & 0.04 & 0.10 & 1.18 & 100.01 \\
\hline $16-1,114-115$ & 49.49 & 1.31 & 14.31 & 10.86 & 0.18 & 7.67 & 12.21 & 2.66 & 0.05 & 0.11 & 1.11 & 99.96 \\
\hline $16-1,133-134$ & 49.23 & 1.34 & 14.29 & 11.16 & 0.18 & 7.64 & 12.02 & 2.47 & 0.05 & 0.11 & 1.18 & 99.67 \\
\hline $16-2,58-60$ & 49.62 & 1.35 & 14.32 & 11.18 & 0.18 & 7.75 & 12.03 & 2.36 & 0.05 & 0.11 & 0.97 & 99.92 \\
\hline $16-3,10-12$ & 49.54 & 1.25 & 14.45 & 10.60 & 0.16 & 8.28 & 11.66 & 2.57 & 0.05 & 0.11 & 1.46 & 100.13 \\
\hline $17-1,64-66$ & 48.26 & 0.97 & 16.12 & 9.53 & 0.17 & 9.48 & 12.29 & 2.08 & 0.02 & 0.09 & 1.39 & 100.40 \\
\hline $17-3,16-18$ & 48.28 & 0.95 & 16.31 & 9.47 & 0.16 & 9.31 & 12.63 & 1.98 & 0.02 & 0.08 & 1.39 & 100.58 \\
\hline $18-4,103-10$ & 48.23 & 0.95 & 16.10 & 9.46 & 0.16 & 9.13 & 12.54 & 1.98 & 0.02 & 0.09 & 1.73 & 100.39 \\
\hline $18-4,140-142$ & 49.05 & 1.72 & 14.89 & 10.27 & 0.15 & 6.97 & 11.87 & 2.70 & 0.13 & 0.19 & 1.11 & 99.05 \\
\hline $19-1,6-8$ & 48.61 & 1.70 & 15.16 & 10.35 & 0.16 & 7.06 & 12.12 & 2.75 & 0.06 & 0.17 & 1.94 & 100.08 \\
\hline $20-1,39-40$ & 48.96 & 1.88 & 14.26 & 12.31 & 0.20 & 7.04 & 11.56 & 2.53 & 0.11 & 0.18 & 0.83 & 99.86 \\
\hline $20-1,144-146$ & 48.88 & 1.91 & 14.79 & 11.66 & 0.20 & 7.04 & 11.55 & 2.51 & 0.06 & 0.19 & 1.25 & 100.04 \\
\hline $21-1,86-88$ & 49.04 & 1.86 & 14.70 & 12.15 & 0.22 & 6.95 & 11.64 & 2.41 & 0.08 & 0.17 & 0.69 & 99.91 \\
\hline $21-2,78-80$ & 49.11 & 1.87 & 14.70 & 12.22 & 0.20 & 6.96 & 11.66 & 2.47 & 0.08 & 0.18 & 0.69 & 100.14 \\
\hline $21-3,53-55$ & 48.58 & 1.78 & 14.59 & 12.01 & 0.22 & 7.23 & 11.81 & 2.38 & 0.06 & 0.18 & 1.18 & 100.02 \\
\hline $22-1,40-42$ & 48.78 & 1,72 & 14.36 & 11.76 & 0.20 & 7.24 & 11.91 & 2.33 & 0.10 & 0.16 & 0.76 & 99.32 \\
\hline $22-2,84-87$ & 49.00 & 1.65 & 14.76 & 11.38 & 0.19 & 6.97 & 12.24 & 2.38 & 0.11 & 0.15 & 1.18 & 100.01 \\
\hline $22-3,38-40$ & 48.32 & 1.69 & 15.13 & 10.62 & 0.17 & 6.63 & 12.00 & 2.53 & 0.05 & 0.16 & 2.22 & 99.52 \\
\hline $22-4,30-32$ & 48.71 & 1.60 & 14.75 & 11.35 & 0.18 & 7.16 & 11.92 & 2.39 & 0.11 & 0.16 & 1.11 & 99.44 \\
\hline $23-1,12-14$ & 48.35 & 1.70 & 15.00 & 11.48 & 0.19 & 6.69 & 12.18 & 2.29 & 0.07 & 0.17 & 1.67 & 99.79 \\
\hline $23-1,100-110$ & 48.85 & 1.59 & 14,61 & 11.39 & 0.19 & 7.61 & 11.90 & 2.45 & 0.04 & 0.14 & 1.18 & 99.95 \\
\hline $23-2,32-35$ & 48.71 & 1.64 & 14.80 & 11.49 & 0.19 & 7.09 & 11.86 & 2.38 & 0.07 & 0.17 & 1.32 & 99.72 \\
\hline $24-2,58-60$ & 48.56 & 2.17 & 13.51 & 13.05 & 0.19 & 6.43 & 10.95 & 2.55 & 0.11 & 0.21 & 0.83 & 98.56 \\
\hline $25-1,3-5$ & 48.96 & 1.27 & 15.70 & 10.77 & 0.19 & 6.87 & 12.51 & 2.34 & 0.06 & 0.12 & 0.97 & 99.76 \\
\hline
\end{tabular}


Table 2. (Continued).

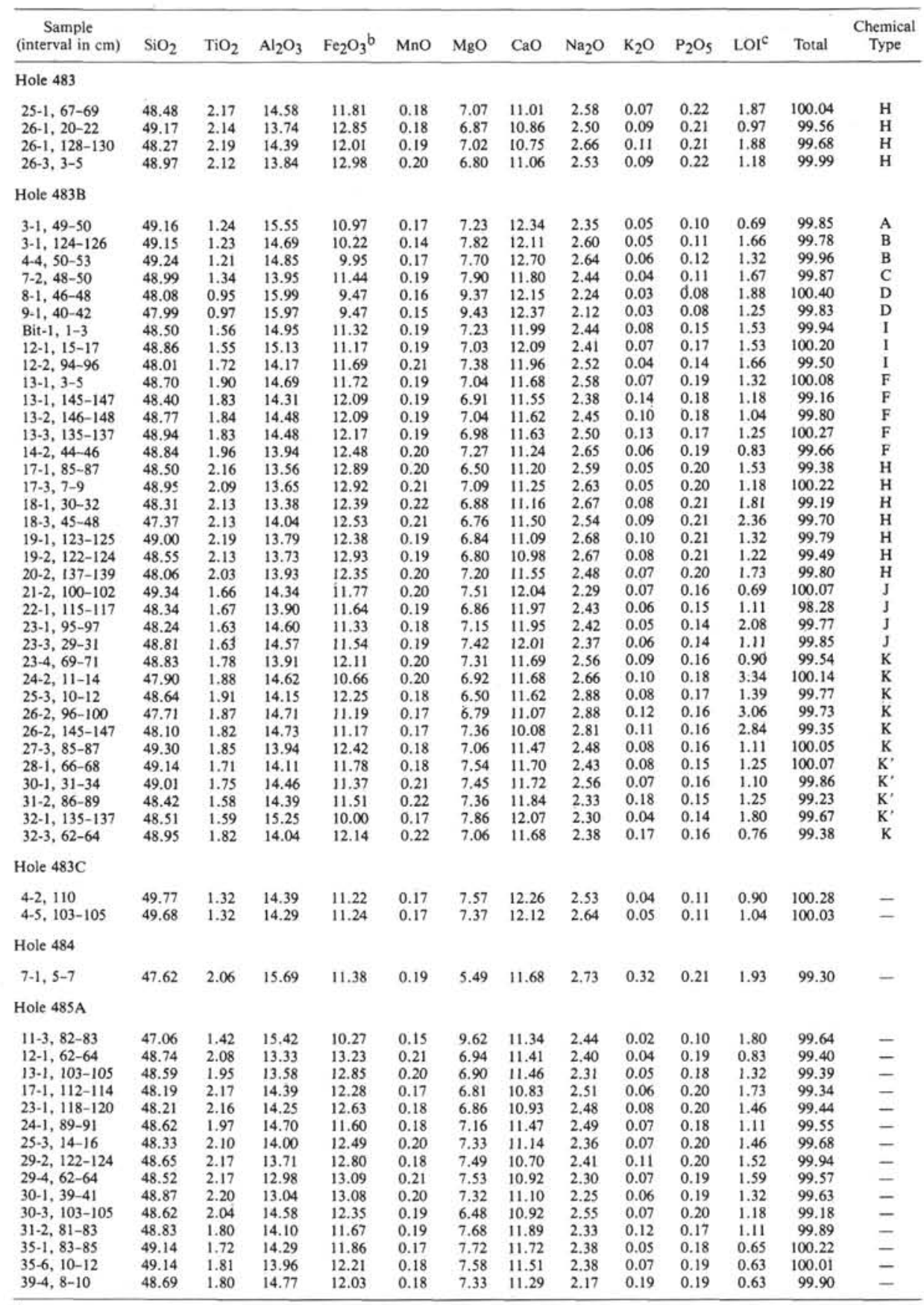

a Values shown in wt.\%.

$\mathrm{c}_{\mathrm{LOI}}=$ loss on ignition at $1050^{\circ} \mathrm{C}$; heated to $110^{\circ} \mathrm{C}$ for $1 \mathrm{hr}$. prior to determination of loss on ignition.

the $\mathrm{Cr}$ and $\mathrm{Ni}$ concentrations $(517$ and $220 \mathrm{ppm}$, respectively), which are similar to the theoretical values for a primitive liquid and to observed concentrations in the most primitive glass samples recovered in the FAMOUS area (Cr: 600 ppm; Ni: 250 ppm; Bougault et al., 1979). Thus the process of fractional crystallization is observed through high partition coefficient elements (such as $\mathrm{Cr}$ and $\mathrm{Ni}$ ), but the observed concentrations of these elements also indicate that some of the liquids have evolved along independent paths in terms of the proportions of olivine and clinopyroxene removed. These observations, together with the absence of a correlation between the degree of fractionation on the one hand and either the distance from the axis or the depth in the crust on the other seems to argue for the existence of several magma chambers rather than a single large magma chamber under the ridge (O'Hara, 1977; Bryan and Thompson, 1979).

It should also be noted that if all of the liquids represented by the basalts in this study had been derived from the same magma chamber, we should observe an inverse correlation between $\mathrm{Mg}, \mathrm{Ni}$, and $\mathrm{Cr}$ on the one hand 
Table 3. Trace element composition of basalts. ${ }^{a}$

\begin{tabular}{|c|c|c|c|c|c|c|c|c|c|c|c|c|c|c|c|c|c|c|c|c|c|c|c|c|c|}
\hline \multirow{2}{*}{$\begin{array}{c}\text { Sample } \\
\text { (interval in } \mathrm{cm} \text { ) }\end{array}$} & \multirow{2}{*}{$\begin{array}{c}\text { Chemical } \\
\text { Type }\end{array}$} & \multirow[b]{2}{*}{$\mathrm{Sc}$} & \multirow[b]{2}{*}{$\mathrm{Ti}$} & \multirow[b]{2}{*}{ v } & \multirow[b]{2}{*}{$\mathrm{Cr}$} & \multirow[b]{2}{*}{$\mathrm{Mn}$} & \multirow[b]{2}{*}{$\mathrm{Fe}$} & \multicolumn{2}{|c|}{ Co } & $\mathrm{N}$ & & & & & & $\mathrm{Zr}$ & & & & & & & & & \\
\hline & & & & & & & & XRF & NAA & XRF & NAA & $\mathrm{Zn}$ & $\mathrm{Rb}$ & $\mathrm{Sr}$ & $\mathrm{Y}$ & XRF & $\mathrm{Nb}$ & Cs & La & Eu & $\mathrm{Tb}$ & $\mathrm{Hf}$ & $\mathrm{Ta}$ & Th & $u$ \\
\hline Hole 482B & & & & & & & & & & & & & & & & & & & & & & & & & \\
\hline $10-7,14-16$ & A & & 10,620 & 335 & 281 & 1781 & 91,350 & 42 & & 65 & & 75 & $<1$ & 107 & 44 & 103 & 2.5 & & & & & & & & \\
\hline $11-1,23-25$ & A & 40 & 10,560 & 344 & 292 & 1549 & 79,730 & 41 & 44 & 74 & 71 & 80 & $<1$ & 111 & 47 & 119 & 3.0 & 0.06 & 3.08 & 1.38 & 0.9 & 3.11 & 0.14 & 0.08 & 0.04 \\
\hline $12-2,135-137$ & A & & 10,440 & 345 & 293 & 1394 & 81,270 & 41 & & 73 & & 70 & $<1$ & 107 & 46 & n.d. & 2,0 & & & & & & & & \\
\hline $14-2,121-124$ & $F$ & & 8,340 & 293 & 260 & 1394 & 77,490 & 43 & & 98 & & 71 & $<1$ & 91 & 37 & 84 & 1.5 & & & & & & & & \\
\hline $15-1,135-139$ & B & & 7,800 & 324 & 192 & 1394 & 76,930 & 44 & & 58 & & 74 & $<1$ & 92 & 37 & 80 & 1.0 & & & & & & & & \\
\hline $15-3,117-119$ & B & & 7,560 & 307 & 184 & 1239 & 76,650 & 41 & & 57 & & 73 & $<1$ & 94 & 35 & 81 & 2.5 & & & & & & & & \\
\hline $16-1,36-38$ & B & & 7,620 & 309 & 176 & 1239 & 76,580 & 43 & & 63 & & 65 & $<1$ & 93 & 35 & 84 & 1.3 & & & & & & & & \\
\hline $17-2,111-113$ & B & 38 & 7,800 & 309 & 187 & 1471 & 79,450 & 43 & 45 & 60 & 55 & 73 & $<1$ & 94 & 36 & 79 & 1.8 & 0.01 & 1.79 & 1.14 & 0.67 & 1.98 & 0.08 & 0.05 & \\
\hline $18-1,18-20$ & B & 41 & 7,800 & 314 & 178 & 1316 & 78,820 & 44 & 47 & 60 & 58 & 73 & $<1$ & 93 & 35 & 83 & 1.5 & 0.04 & 2.14 & 1.06 & 0.71 & 2.13 & 0.09 & 0.05 & 0.06 \\
\hline $18-2,99-101$ & $\mathrm{D}^{\prime}$ & & 9,240 & 339 & 197 & 1471 & 82,670 & 43 & & 64 & & 79 & $<1$ & 90 & 43 & 101 & 1.0 & & 2.38 & & & & & & 0.03 \\
\hline $19-1,96-100$ & C & & 13,200 & 464 & 150 & 929 & 80,920 & 47 & & 51 & & 79 & $<1$ & 119 & 50 & 151 & 2.0 & & & & & & & & \\
\hline $20-3,24-26$ & $\mathrm{D}^{\prime}$ & & 9,240 & 371 & 199 & 1239 & 69,790 & 43 & & 67 & & 81 & $<1$ & 97 & 43 & 103 & 1.3 & & & & & & & & \\
\hline $21-1,62-64$ & E & & 11,280 & 354 & 197 & 1239 & 81,900 & 40 & & 67 & & 69 & $<1$ & 112 & 50 & 130 & 2.0 & & & & & & & & \\
\hline $21-3,74-76$ & D"' & 39 & 9,660 & 317 & 241 & 1316 & 74,550 & 39 & 42 & 62 & 62 & 74 & $<1$ & 126 & 41 & 116 & 1.5 & 0.01 & 2.97 & 1.35 & 0.83 & 2.88 & 0.10 & 0.06 & 0.06 \\
\hline $22-2,34-36$ & $\mathrm{D}^{\prime \prime \prime}$ & & 9,360 & 305 & 228 & 1239 & 74,480 & 38 & & 61 & & 67 & $<1$ & 125 & 41 & 116 & 1.5 & & & & & & & & \\
\hline $22-4,34-36$ & $\mathrm{D}^{\prime \prime \prime}$ & & 9,060 & 296 & 263 & 1239 & 72,590 & 39 & & 71 & & 63 & $<1$ & 125 & 40 & 106 & 1.0 & & & & & & & & \\
\hline $24-1,93-95$ & $\mathrm{D}^{\prime \prime}$ & 42 & 9,780 & 385 & 193 & 1162 & 71,540 & 46 & 46 & 63 & 66 & 80 & $<i$ & 96 & 44 & 108 & 2.0 & 0.08 & 2.50 & 1.09 & 0.84 & 2.75 & 0.10 & 0.06 & 0.23 \\
\hline Hole $482 \mathrm{C}$ & & & & & & & & & & & & & & & & & & & & & & & & & \\
\hline $9-1,121-123$ & A & & 10,620 & 361 & 277 & 1549 & 81,060 & 40 & & 67 & & 85 & $<1$ & 110 & 48 & 125 & 2.5 & & & & & & & & \\
\hline $10-2,73-75$ & A & 39 & 10,260 & 353 & 288 & 1471 & 79,870 & 42 & 43 & 72 & 72 & 73 & $<1$ & 106 & 45 & 121 & 1.5 & 0.6 & 2.9 & 1.35 & 0.89 & 3.1 & 0.13 & 0.09 & 0.04 \\
\hline $11-1,115-117$ & A & 40 & 10,680 & 365 & 323 & 1316 & 79,520 & 43 & 45 & 81 & 77 & 70 & $<1$ & 114 & 48 & 127 & 3.0 & 0.06 & 2.9 & 1.42 & 0.91 & 3.05 & 0.14 & 0.09 & \\
\hline $11-1,138-139$ & A & & 10,380 & 344 & 357 & 1316 & 77,560 & 41 & & 84 & & 70 & $<1$ & 111 & 48 & 122 & 2.5 & & & & & & & & \\
\hline $11-2,16-18$ & $\mathrm{~F}$ & & 7,920 & 292 & 361 & 1162 & 74,060 & 38 & & 79 & & 40 & $<1$ & 92 & 36 & 88 & 0.5 & & & & & & & & \\
\hline $11-2,21-24$ & F & & 7,140 & 261 & 376 & 1007 & 80,570 & 37 & & 80 & & 26 & $<1$ & 84 & 30 & 75 & 1.0 & & & & & & & & \\
\hline $11-3,141-143$ & A & & 10,740 & 329 & 294 & 1394 & 8,160 & 41 & & 72 & & 67 & $<1$ & 113 & 48 & 120 & 1.5 & & & & & & & & \\
\hline $12-1,11-13$ & B & & 7,800 & 310 & 182 & 1397 & 78,400 & 42 & & 59 & & 75 & $<1$ & 93 & 35 & 81 & 1.0 & & & & & & & & \\
\hline $12-2,95-97$ & B & & 7,800 & 305 & 172 & 1397 & 79,310 & 41 & & 58 & & 69 & $<1$ & 94 & 36 & 87 & 2.0 & & & & & & & & \\
\hline $13-1,22-24$ & B & 39 & 7,800 & 325 & 177 & 1162 & $.73,290$ & 42 & 44 & 55 & 57 & 65 & $<1$ & 93 & 36 & 80 & 2.0 & 0.2 & 1.84 & 1.03 & 0.68 & 2.05 & 0.09 & 0.06 & 0.20 \\
\hline $13-1,125-127$ & B & & 7,740 & 311 & 175 & 1316 & 77,910 & 40 & & 56 & & 71 & $<1$ & 93 & 35 & 76 & 2.5 & & & & & & & & \\
\hline $13-3,108-110$ & B & & 7,800 & 307 & 172 & 1394 & 78,890 & 43 & & 57 & & 70 & $<1$ & 93 & 36 & 78 & 2.0 & & & & & & & & \\
\hline $14-1,40-42$ & B & & 7,680 & 287 & 163 & 1316 & 77,420 & 40 & & 54 & & 64 & $<1$ & 94 & 36 & 81 & 1.0 & & & & & & & & \\
\hline $14-4,127-129$ & B & & 7,740 & 306 & 195 & 1316 & 78,540 & 41 & & 57 & & 59 & $<1$ & 92 & 36 & 75 & 1.5 & & & & & & & & \\
\hline $15-1,44-46$ & B & & 7,620 & 305 & 180 & 1394 & 78,330 & 40 & & 58 & & 68 & $<1$ & 92 & 35 & 80 & 2.0 & & & & & & & & \\
\hline $15-2,132-134$ & B & & 7,620 & 292 & 170 & 1316 & 77,210 & 41 & & 59 & & 72 & $<1$ & 92 & 35 & 75 & 1.0 & & & & & & & & \\
\hline $15-3,97-99$ & B & & 7,620 & 307 & 174 & 1394 & 78,120 & 41 & & 56 & & 65 & $<1$ & 94 & 35 & 74 & 1.5 & & & & & & & & \\
\hline $15-4,119-121$ & B & & 7,560 & 305 & 191 & 1394 & 77,700 & 40 & & 60 & & 65 & $<1$ & 92 & 34 & 82 & 2.0 & & & & & & & & \\
\hline Hole 482D & & & & & & & & & & & & & & & & & & & & & & & & & \\
\hline $8-1,30$ & A & & 10,740 & 340 & 306 & 1936 & 76,30 & 38 & & 60 & & 75 & 4.4 & 104 & 44 & 111 & 2.0 & & & & & & & & \\
\hline $8-1,109-111$ & A & & 10,500 & 355 & 268 & 1471 & 80,570 & 43 & & 70 & & 74 & $<1$ & 111 & 46 & 119 & 2.0 & & & & & & & & \\
\hline $9-1,48-50$ & A & & 10,680 & 357 & 301 & 2549 & 81,130 & 42 & & 73 & & 83 & $<1$ & 107 & 46 & 120 & 2.5 & & & & & & & & \\
\hline $9-1,50-52$ & A & 41 & 10,800 & 357 & 293 & 1549 & 82,880 & 43 & 44 & 71 & 71 & 72 & $<1$ & 109 & 48 & 124 & 3.0 & 0.11 & 3.1 & 1.5 & 0.86 & 3.36 & 0.14 & 0.1 & 0.05 \\
\hline $10-2,53-55$ & A & & 10,560 & 341 & 288 & 1471 & 81,200 & 40 & & 71 & & 79 & $<1$ & 108 & 47 & 127 & 2.5 & & & & & & & & \\
\hline $10-2,109-112$ & A & & 10,620 & 346 & 287 & 1394 & 79,800 & 41 & & 73 & & 76 & $<1$ & 106 & 46 & 122 & 2.0 & & & & & & & & \\
\hline $10-3,14-16$ & E & 42 & 11,400 & 360 & 265 & 1471 & 83,440 & 42 & 45 & 65 & 66 & 80 & $<1$ & 108 & 51 & 133 & 1.5 & 0.05 & 3.3 & 1.31 & 1.07 & 3.52 & 0.15 & 0.12 & \\
\hline $10-3,101-103$ & E & 38 & 8,460 & 306 & 279 & 1394 & 77,350 & 43 & 46 & 99 & 98 & 73 & $<1$ & 91 & 38 & 91 & 1.5 & 0.08 & 2.26 & 1.23 & 0.75 & 2.38 & 0.09 & 0.06 & 0.23 \\
\hline $11-1,135-137$ & $\mathbf{F}$ & 36 & 8,460 & 307 & 266 & 1394 & 77,700 & 44 & 44 & 86 & 87 & 77 & $<1$ & 94 & 37 & 91 & 1.5 & 0.06 & 2.0 & 1.10 & 0.73 & 2.23 & 0.10 & 0.07 & \\
\hline $12-1,105-107$ & $\mathbf{F}$ & & 8,340 & 303 & 253 & 1394 & 78,190 & 42 & & 96 & & 74 & $<1$ & 97 & 38 & 84 & 3.0 & & & & & & & & \\
\hline $12-2,50-52$ & B & & 7.740 & 316 & 198 & 1394 & 78,680 & 43 & & 63 & & 73 & $<1$ & 93 & 36 & 78 & 3.0 & & & & & & & & \\
\hline $12-2,82-84$ & B & & 7,740 & 317 & 188 & 1316 & 78,190 & 44 & & 63 & & 66 & $<1$ & 91 & 35 & 79 & 2.0 & & & & & & & & \\
\hline $12-3,118-120$ & B & 38 & 7,620 & 293 & 164 & 1316 & 77,420 & 41 & 45 & 59 & 57 & 65 & $<1$ & 95 & 35 & 83 & 2.5 & & 2.05 & 0.98 & 0.68 & 2.21 & 0.08 & 0.04 & \\
\hline $13-1,127-129$ & B & 40 & 8,040 & 340 & 188 & 1239 & 73,570 & 43 & 45 & 64 & 57 & 79 & $<1$ & 99 & 36 & 80 & 1.0 & 0.03 & 1.90 & 0.98 & 0.68 & 2.09 & 0.09 & 0.05 & \\
\hline $13-3,4-6$ & B & & 7,860 & 314 & 163 & 1316 & 78,120 & 43 & & 61 & & 70 & $<1$ & 95 & 37 & 85 & 2.5 & & & & & & & & \\
\hline
\end{tabular}

Hole $482 \mathrm{~F}$

4-3, 131-137

$\begin{array}{llllll}11,700 & 360 & 212 & 1471 & 85,960\end{array}$

Hole 483

\begin{tabular}{|c|c|c|c|c|c|c|c|}
\hline $13-4,17-19$ & A & & 7,620 & 311 & 174 & 1,239 & 75,950 \\
\hline $14-1,35-37$ & B & 37 & 7,080 & 267 & 369 & 1,781 & 73,780 \\
\hline $14-2,12-14$ & B & & 7,200 & 275 & 373 & 1.239 & 72,940 \\
\hline $15-1,21-24$ & B & & 7,140 & 256 & 380 & 1,316 & 73,430 \\
\hline $15-2,96-98$ & B & & 7,140 & 261 & 378 & 1.239 & 72,030 \\
\hline $15-3,7-9$ & C & & 8,220 & 298 & 241 & 1,316 & 76,090 \\
\hline $16-1,114-115$ & C & & 7,860 & 288 & 258 & 1,394 & 76,020 \\
\hline $16-1,133-134$ & c & & 8,040 & 291 & 242 & 1,394 & 78,120 \\
\hline $16-2,58-60$ & C & & 8,100 & 304 & 238 & 1,394 & 78,260 \\
\hline $16-3,10-12$ & & & 7,500 & 300 & 320 & 1,239 & 74,200 \\
\hline $17-1,64-66$ & D & & 5,820 & 217 & 358 & 1,316 & 66,710 \\
\hline $17-3,16-18$ & D & & 5,700 & 208 & 333 & 1.239 & 66,290 \\
\hline $18-4,103-105$ & D & & 5,700 & 215 & 348 & 1.239 & 66,220 \\
\hline $18-4,140-142$ & E & & 10,320 & 288 & 300 & 1,161 & 71,890 \\
\hline $19-1,6-8$ & $\bar{E}$ & & 10,200 & 303 & 296 & 1,239 & 72,450 \\
\hline $20-1,39-40$ & $\mathrm{~F}$ & & 11,280 & 354 & 188 & 1,549 & 86,170 \\
\hline $20-1,144-146$ & $\mathrm{~F}$ & & 11,460 & 385 & 192 & 1,549 & 81,620 \\
\hline $21-1,86-88$ & $F$ & & 11,160 & 372 & 198 & 1,704 & 85,050 \\
\hline $21-2,78-80$ & $\mathrm{~F}$ & & 11,220 & 370 & 205 & 1,549 & 85,540 \\
\hline $21-3,53-55$ & G? & & 10,680 & 362 & 222 & 1,704 & 84,070 \\
\hline $22-1,40-42$ & $\mathrm{~F}^{\prime}$ & & 10,320 & 337 & 274 & 1.549 & 82,320 \\
\hline $22-2,84-87$ & $\mathrm{~F}^{\prime}$ & & 9,990 & 340 & 297 & 1,471 & 79,660 \\
\hline $22-3,38-40$ & $\mathrm{~F}^{\prime}$ & & 10,140 & 350 & 316 & 1,316 & 74,340 \\
\hline $22-4,30-32$ & $F^{\prime}$ & & 9,600 & 321 & 283 & 1,394 & 79,450 \\
\hline $23-1,12-14$ & $\mathrm{~F}^{\prime}$ & & 10,200 & 335 & 248 & 1,471 & 80,360 \\
\hline $23-1,108-110$ & $\mathrm{~F}^{*}$ & & 9,540 & 311 & 278 & 1,471 & 80,360 \\
\hline $23-2,32-35$ & $\mathrm{~F}^{\prime}$ & 39 & 9,840 & 333 & 258 & 1,471 & 80,430 \\
\hline $24-2,58-60$ & $\mathrm{H}$ & 41 & 13,020 & 390 & 162 & 1,471 & 91,350 \\
\hline $25-1,3-5$ & A & 38 & 7,620 & 293 & 180 & 1,471 & 75,390 \\
\hline $25-1,67-69$ & $\mathrm{H}$ & & 13,020 & 440 & 169 & 1,394 & 82,670 \\
\hline $26-1,20-22$ & $\mathrm{H}$ & & 12,840 & 395 & 163 & 1,394 & 89,950 \\
\hline $26-1,128-130$ & H & 43 & 13,140 & 430 & 182 & 1,471 & 84,070 \\
\hline $26-3,3-5$ & $\mathrm{H}$ & & 12,720 & 386 & 155 & 1,549 & 90,860 \\
\hline
\end{tabular}

\begin{tabular}{|c|c|c|c|c|c|c|c|c|c|c|c|c|c|c|c|c|c|}
\hline 40 & & 70 & & 73 & $<1$ & 993 & 34 & 74 & 1 & & & & & & & & \\
\hline 42 & 45 & 69 & 65 & 80 & $<1$ & 110 & 30 & 78 & 2.5 & 0.14 & 1.72 & 1.04 & 0.59 & 1.84 & 0.10 & 0.04 & \\
\hline 43 & & 73 & & 62 & $<1$ & 114 & 31 & 75 & 2 & & & & & & & & \\
\hline 42 & & 71 & & 56 & $<1$ & 113 & 31 & 77 & 1 & & & & & & & & \\
\hline 40 & & 68 & & 59 & $<1$ & 114 & 30 & 78 & 2 & & & & & & & & \\
\hline 44 & & 57 & & 73 & $<1$ & 97 & 36 & 79 & 1.5 & & & & & & & & \\
\hline 43 & & 58 & & 62 & $<1$ & 94 & 34 & 75 & 1.5 & & & & & & & & \\
\hline 42 & & 57 & & 70 & $<1$ & 94 & 36 & 75 & 1.5 & & & & & & & & \\
\hline 45 & & 57 & & 74 & $<1$ & 94 & 36 & 80 & 1.5 & & & & & & & & \\
\hline 44 & & 61 & & 69 & $<1$ & 103 & 33 & 72 & 1.5 & & & & & & & & \\
\hline 44 & & 107 & & 52 & $<1$ & 97 & 26 & 57 & 0.5 & & & & & & & & \\
\hline 44 & & 106 & & 51 & $<1$ & 134 & 25 & 61 & 0.5 & & & & & & & & \\
\hline 43 & & 112 & & 58 & $<1$ & 101 & 25 & 60 & 0.0 & & & & & & & & \\
\hline 41 & & 89 & & 73 & $<1$ & 166 & 41 & 124 & 3 & & & & & & & & \\
\hline 42 & & 88 & & 73 & $<1$ & 166 & 41 & 128 & 3 & & & & & & & & \\
\hline 43 & & 68 & & 86 & $<1$ & 101 & 49 & 132 & 2 & & & & & & & & \\
\hline 45 & & 66 & & 90 & $<1$ & 106 & 51 & 138 & 3.5 & & & & & & & & \\
\hline 43 & & 67 & & 91 & $<1$ & 101 & 49 & 131 & 3 & & & & & & & & \\
\hline 43 & & 67 & & 88 & $<1$ & 101 & so & 134 & 2.5 & & & & & & & & \\
\hline 43 & & 68 & & 85 & $<1$ & 100 & 47 & 124 & 2.5 & & & & & & & & \\
\hline 42 & & 78 & & 83 & $<1$ & 98 & 46 & 123 & 2 & & & & & & & & \\
\hline 42 & & 82 & & 81 & $<1$ & 96 & 45 & 116 & 3 & & & & & & & & \\
\hline 44 & & 89 & & 86 & $<1$ & 102 & 46 & 126 & 2.5 & & & & & & & & \\
\hline 41 & & 91 & & 78 & 1 & 99 & 42 & 105 & 2.5 & & & & & & & & \\
\hline 41 & & 77 & & 81 & $<1$ & 103 & 45 & 120 & 2 & & & & & & & & \\
\hline 41 & & 98 & & 68 & $<1$ & 99 & 43 & 113 & 1.5 & & & & & & & & \\
\hline 42 & 44 & 77 & 82 & 80 & $<1$ & 101 & 44 & 118 & 2.5 & 0.05 & 3.1 & 1.06 & 0.81 & 3.12 & 0.16 & 0.14 & \\
\hline 44 & 46 & 70 & 71 & 90 & $<1$ & 105 & 54 & 155 & 3 & & 4.5 & 1.38 & 1.09 & 3.94 & 0.23 & 0.19 & 0.07 \\
\hline 40 & 44 & 64 & 64 & 70 & $<1$ & 89 & 33 & 71 & 1.5 & 0.04 & 1.77 & 0.95 & 0.60 & 2.04 & 0.08 & 0.06 & 0.04 \\
\hline 48 & & 77 & & 98 & $<1$ & 114 & 56 & 156 & 4 & & & & & & & & \\
\hline 42 & & 68 & & 88 & $<1$ & 104 & 55 & 158 & 4 & & & & & & & & \\
\hline 46 & 47 & 67 & 70 & 95 & $<1$ & 109 & 56 & 156 & 3.5 & 0.04 & 4.3 & 1.59 & 1.15 & 3.98 & 0.22 & 0.19 & 0.28 \\
\hline 43 & & 63 & & 87 & $<1$ & 104 & 55 & 150 & 3.5 & & & & & & & & \\
\hline
\end{tabular}


Table 3. (Continued).

\begin{tabular}{|c|c|c|c|c|c|c|c|c|c|c|c|c|c|c|c|c|c|c|c|c|c|c|c|c|c|}
\hline \multirow{2}{*}{$\begin{array}{c}\text { Sample } \\
\text { (interval in } \mathrm{cm} \text { ) }\end{array}$} & \multirow{2}{*}{$\begin{array}{l}\text { Chemical } \\
\text { Type }\end{array}$} & \multirow[b]{2}{*}{$\mathrm{Sc}$} & \multirow[b]{2}{*}{$\mathrm{Ti}$} & \multirow[b]{2}{*}{$\mathrm{v}$} & \multirow[b]{2}{*}{$\mathrm{Cr}$} & & & & & & ii & & & & & $\mathrm{Zr}$ & & & & & & & & & \\
\hline & & & & & & $\mathrm{Mn}$ & $\mathrm{Fe}$ & XRF & NAA & XRF & NAA & $\mathrm{Zn}$ & $\mathrm{Rb}$ & Sr & $Y$ & $\mathrm{XRF}$ & $\mathrm{Nb}$ & Cs & $\mathrm{La}$ & $\mathrm{Eu}$ & $\mathrm{Tb}$ & $\mathrm{Hf}$ & $\mathrm{Ta}$ & Th & U \\
\hline Hole 483B & & & & & & & & & & & & & & & & & & & & & & & & & \\
\hline $3-1,49-50$ & $\mathrm{~A}$ & 36 & 7,440 & 290 & 176 & 1,316 & 76,790 & 40 & 44 & 65 & 62 & 68 & $<1$ & 88 & 33 & 67 & 1.5 & 0.06 & 1.7 & 1.09 & 0.66 & 1.97 & 0.08 & 0.05 & 0.04 \\
\hline $3-1,124-126$ & B & & 7.380 & 280 & 370 & 1,084 & 71,540 & 42 & & 71 & 68 & 58 & $<i$ & 116 & 31 & 75 & 2 & & & & & & & & \\
\hline $4-4,50-53$ & B & & 7,260 & 270 & 381 & 1,316 & 69,650 & 43 & & 72 & & 51 & $<1$ & 114 & 32 & 75 & 2 & & & & & & & & \\
\hline $7-2,48-50$ & c & & 8,040 & 281 & 243 & 1,471 & 80,080 & 42 & & 60 & & 65 & $<1$ & 105 & 35 & 78 & 2 & & & & & & & & \\
\hline $8-1,46-48$ & D & & 5,700 & 207 & 351 & 1,239 & 66,290 & 44 & & 115 & & 55 & $<1$ & 93 & 26 & 62 & 1 & & & & & & & & \\
\hline $9-1,40-42$ & D & & 5,820 & 199 & 336 & 1,162 & 66,290 & 42 & & 107 & & 50 & $<1$ & 98 & 24 & 58 & 0.5 & & & & & & & & \\
\hline Bit-1, 1-3 & 1 & & 9,360 & 307 & 209 & 1,471 & 79,240 & 43 & & 74 & & 72 & $<i$ & 116 & 40 & 104 & 3.5 & & & & & & & & \\
\hline $12-1,15-17$ & I & & 9,300 & 312 & 221 & 1,471 & 78,190 & 42 & & 75 & & 74 & $<1$ & 117 & 40 & 106 & 2.5 & & & & & & & & \\
\hline $12-2,94-96$ & 1 & 43 & 10,320 & 355 & 213 & 1,626 & 81,830 & 43 & 46 & 66 & 66 & 81 & $<1$ & 115 & 44 & 113 & 4 & 0.02 & 2.9 & 1.3 & 0.93 & 2.95 & 0.15 & 0.11 & \\
\hline $13-1,3-5$ & F & & 11,400 & 379 & 211 & 1,471 & 82,040 & 45 & & 71 & & 88 & $<1$ & 106 & 51 & 135 & 2.5 & & & & & & & & \\
\hline $13-1,145-147$ & F & & 10,980 & 348 & 209 & 1,471 & 84,630 & 43 & & 75 & & 77 & $<1$ & 102 & 48 & 135 & 2 & & & & & & & & \\
\hline $13-2,146-148$ & F & & 11,040 & 363 & 214 & 1,471 & 84,630 & 42 & & 67 & & 84 & $<i$ & 102 & 50 & 140 & 3 & & & & & & & & \\
\hline $13-3,135-137$ & $\mathrm{~F}$ & 41 & 10,980 & 354 & 210 & 1.471 & 85,190 & 41 & 45 & 69 & 72 & 83 & 1 & 105 & 50 & 136 & 3.5 & 0.06 & 3.7 & 1.52 & 1.05 & 3.62 & 0.19 & 0.16 & \\
\hline $14-2,44-46$ & F & & 11,760 & 390 & 216 & 1,549 & 87,360 & 45 & & 66 & & 88 & $<1$ & 102 & 52 & 140 & 4.5 & & & & & & & & \\
\hline $17-1,85-87$ & $\mathrm{H}$ & & 12,960 & 387 & 166 & 1,549 & 90,230 & 44 & & 64 & & 87 & $<1$ & 107 & 55 & 156 & 3.5 & & & & & & & & \\
\hline $17-3,7-9$ & H & & 12,540 & 385 & 176 & 1,626 & 90,440 & 44 & & 65 & & 83 & $<i$ & 105 & 54 & 153 & 3.5 & & & & & & & & \\
\hline $18-1,30-32$ & $\mathrm{H}$ & & 12,780 & 412 & 165 & 1,704 & 86,730 & 43 & & 62 & & 87 & $<1$ & 107 & 55 & 157 & 4 & & & & & & & & \\
\hline $18-3,45-48$ & $\mathrm{H}$ & & 12,780 & 425 & 171 & 1,626 & 87,710 & 46 & & 65 & & 92 & $<1$ & 108 & 56 & 156 & 4.5 & & & & & & & & \\
\hline $19-1,123-125$ & $\mathrm{H}$ & & 13,140 & 410 & 158 & 1,471 & 86,660 & 44 & & 59 & & 91 & $<1$ & 109 & 58 & 161 & 4 & & & & & & & & \\
\hline $19-2,122-124$ & $\mathrm{H}$ & & 12,780 & 401 & 166 & 1,471 & 90,510 & 44 & & 65 & & 96 & $<1$ & 105 & 56 & 155 & 5 & & & & & & & & \\
\hline $20-2,137-139$ & $\mathrm{H}$ & & 12,180 & 403 & 249 & 1,549 & 86,450 & 44 & & 69 & & 91 & $<i$ & 100 & 53 & 147 & 3 & & & & & & & & \\
\hline $21-2,100-102$ & J & & 9,960 & 351 & 225 & 1,549 & 82,390 & 44 & & 63 & & 83 & $<1$ & 108 & 42 & 108 & 2 & & & & & & & & \\
\hline $22-1,115-117$ & J & & 10,020 & 340 & 215 & 1,471 & 81,480 & 44 & & 59 & & 76 & $<1$ & 107 & 41 & 107 & 2 & & & & & & & & \\
\hline $23-1,95-97$ & $\mathrm{~J}$ & & 9,780 & 334 & 218 & 1,394 & 79,310 & 43 & & 54 & & 78 & $<i$ & 108 & 41 & 106 & 2 & & & & & & & & \\
\hline $23-3,29-31$ & $\mathbf{J}$ & 38 & 9,780 & 336 & 218 & 1,471 & 80,780 & 44 & 44 & 69 & 74 & 79 & $<1$ & 109 & 40 & 106 & 2.5 & 0.04 & 3.3 & 0.99 & 0.80 & 2.73 & 0.15 & 0.11 & 0.18 \\
\hline $23-4,69-71$ & $\mathrm{~K}$ & & 10,680 & 361 & 205 & 1,549 & 84,770 & 45 & & 67 & & 81 & $<1$ & 109 & 45 & 118 & 2.5 & & & & & & & & \\
\hline $24-2,11-14$ & $\mathrm{~K}$ & & 11,280 & 394 & 195 & 1,549 & 74,620 & 46 & & 68 & & & $<1$ & 119 & 48 & 121 & 2.5 & & & & & & & & \\
\hline $25-3,10-12$ & K & & 11.460 & 359 & 154 & 1,394 & 85,740 & 43 & & 54 & & 85 & $<1$ & 114 & 48 & 130 & 2.5 & & & & & & & & \\
\hline $26-2,96-100$ & $\mathrm{~K}$ & & 11,220 & 390 & 197 & 1,316 & 78,330 & 46 & & 66 & & 92 & $<1$ & 119 & 47 & 121 & 3 & & & & & & & & \\
\hline $26-2,145-147$ & $\mathrm{~K}$ & & 10,920 & 379 & 198 & 1,316 & 78,190 & 48 & & 68 & & 85 & i & 115 & 46 & 117 & 3.5 & & & & & & & & \\
\hline $27-3,85-87$ & $\mathrm{~K}$ & & 11,100 & 353 & 166 & 1,394 & 86,940 & 43 & & 61 & & 84 & $<1$ & 112 & 48 & 126 & 3 & & & & & & & & \\
\hline $28-1,66-68$ & $\mathrm{~K}^{\prime}$ & & 10,260 & 332 & 233 & 1,394 & 82,460 & 41 & & 80 & & 68 & $i$ & 109 & 45 & 117 & 3.5 & & & & & & & & \\
\hline $30-1,31-34$ & $\mathrm{~K}^{\prime}$ & 39 & 10,500 & 359 & 265 & 1,626 & 79,590 & 45 & 47 & 109 & 111 & 83 & $<i$ & 112 & 45 & 118 & 3 & & 3.7 & 1.47 & 0.9 & 3.05 & 0.18 & 0.15 & 0.34 \\
\hline $31-2,86-89$ & $\mathrm{~K}^{\prime}$ & & 9,480 & 321 & 358 & 1,703 & 80,570 & 46 & & 128 & & 74 & 2.7 & 110 & 41 & 99 & 3.5 & & & & & & & & \\
\hline $32-1,135-137$ & K' & 38 & 9,540 & 333 & 354 & 1,316 & 70,000 & 44 & 48 & 123 & 125 & 76 & $<1$ & 121 & 41 & 110 & 2 & & 3.2 & 1.12 & 0.75 & 2.66 & 0.17 & 0.19 & 0.13 \\
\hline $32-3,62-64$ & $K$ & & 10,920 & 374 & 185 & 1,703 & 84,980 & 46 & & 68 & & 93 & 2.9 & 106 & 47 & 122 & 2 & & & & & & & & \\
\hline Hole $483 \mathrm{C}$ & & & & & & & & & & & & & & & & & & & & & & & & & \\
\hline $4-2,110$ & & 41 & 7,920 & 298 & 179 & 1,316 & 78,540 & 42 & 48 & 67 & 60 & 65 & $<1$ & 109 & 34 & 89 & 2 & 0.05 & 2.04 & 1.24 & 0.7 & 2.18 & 0.08 & 0.05 & \\
\hline $4-5,103-105$ & & & 7,920 & 288 & 169 & 1,316 & 78,680 & 43 & & 70 & & 64 & $<1$ & 108 & 34 & 84 & 1 & & & & & & & & \\
\hline Hole $484 \mathrm{~A}$ & & & & & & & & & & & & & & & & & & & & & & & & & \\
\hline $7 \cdot 1,5-7$ & & 46 & 12,360 & 320 & 275 & 1,471 & 79,660 & 41 & 44 & 101 & 102 & 86 & 2.2 & 161 & 47 & 140 & 7 & 0.08 & 6.5 & 1.69 & 1.0 & 3.76 & 0.58 & 0.45 & 0.73 \\
\hline Hole $485 \mathrm{~A}$ & & & & & & & & & & & & & & & & & & & & & & & & & \\
\hline $11-3,82-83$ & & 36 & 8,520 & 244 & 517 & 1,16 & 71,8 & 47 & 49 & 220 & 230 & 62 & $<\mathrm{I}$ & 135 & 35 & 90 & 1.5 & 20 & 2.5 & 1.28 & 0.65 & 2.37 & 0.07 & & 0.05 \\
\hline $12-1,62-64$ & & 42 & 12,480 & 432 & 167 & 1,626 & 92,610 & 46 & 45 & 69 & 62 & 92 & $<1$ & 90 & 57 & 134 & 3.0 & & 3.5 & 1.62 & 1.07 & 3.72 & 0.12 & 0.05 & \\
\hline $13-1,103-105$ & & 41 & 11.700 & 408 & 177 & 1,549 & 89.950 & 44 & 46 & 76 & 72 & 90 & $<1$ & 107 & 53 & 132 & 2.0 & 0.7 & 2.8 & 1.70 & 1.10 & 3.42 & 0.12 & 0.05 & \\
\hline $17-1,112-114$ & & 43 & 13,020 & 426 & 252 & 1,316 & 85,960 & 46 & 46 & 101 & 95 & 92 & $<1$ & 105 & 55 & 151 & 3.5 & 0.12 & 3.5 & 1.55 & 1.14 & 4.00 & 0.16 & 0.12 & \\
\hline $23-1,118-120$ & & & 12,960 & 381 & 212 & 1,394 & 88,410 & 43 & & 84 & & 80 & $<1$ & 109 & 56 & 145 & 3.4 & & & & & & & & \\
\hline $24-1,89-91$ & & & 11,820 & 385 & 276 & 1,394 & 81,200 & 42 & & 90 & & 74 & $<1$ & 105 & 51 & 136 & 3.0 & & & & & & & & \\
\hline $25-3,14-16$ & & 42 & 12,600 & 384 & 230 & 1.549 & 87,430 & 43 & 44 & 91 & 86 & 82 & $<1$ & 155 & 55 & 144 & 2.0 & 0.30 & 3.5 & 1.6 & 1.18 & 3.88 & 0.18 & 0.13 & \\
\hline $29-2,122-124$ & & & 13.020 & 390 & 267 & 1,394 & 89,600 & 43 & & 91 & & 85 & 1.9 & 94 & 57 & 142 & 2.5 & & & & & & & & \\
\hline $29-4,62-64$ & & 46 & 13,020 & 419 & 294 & 1,626 & 91,630 & 42 & 45 & 65 & 62 & 88 & 1.0 & 94 & 57 & 143 & 2.0 & 0.17 & 3.95 & 1.62 & 1.16 & 3.83 & 0.18 & 0.11 & \\
\hline $30-1,39-41$ & & & 13,200 & 411 & 335 & 1,549 & 91,560 & 40 & & 59 & & 87 & $<1$ & 97 & 57 & 142 & 2.0 & & & & & & & & \\
\hline $30-3,103-105$ & & 38 & 12,240 & 348 & 266 & 1,471 & 86,450 & 40 & 42 & 75 & 78 & 74 & $<1$ & 106 & 56 & 151 & 1.0 & 0.11 & 3.60 & 1.41 & 1.09 & 3.81 & 0.17 & 0.13 & 0.03 \\
\hline $31-2,81-83$ & & 43 & 10,800 & 351 & 458 & 1,471 & 81,690 & 40 & 44 & 102 & 105 & 77 & 2 & 96 & 49 & 119 & 4.5 & 0.26 & 3.2 & 1.26 & 0.87 & 3.22 & 0.14 & 0.01 & \\
\hline $35-1,83-85$ & & & 10,320 & 337 & 262 & 1,316 & 83,020 & 41 & & 67 & & & $<1$ & 99 & 45 & 108 & 1.7 & & & & & & & & \\
\hline $35-6,10-12$ & & & 10,860 & 347 & 253 & 1,394 & 85,470 & 42 & & 74 & & & $<1$ & 98 & 47 & 110 & 2.4 & & & & & & & & \\
\hline $39-4,8-10$ & & & 10,800 & 342 & 210 & 1,394 & 84,210 & 44 & & & & & $<1$ & 100 & 46 & 112 & 1.4 & & & & & & & & \\
\hline
\end{tabular}

Note: $\mathrm{XRF}=\mathrm{X}$-ray fluorescence; $\mathrm{NAA}=$ neutron activation analysis.

a values shown in ppm.

and $\mathrm{Ti}$ and the hygromagmaphile elements on the other: this is obviously not the case. The absence of such a correlation was interpreted by Rhodes et al. (1979) to result from the mixing of a new primitive liquid with differentiated liquids. Such an explanation is possible, but it cannot apply to large, long-lived magma chambers (Bryan and Thompson, 1979) which are continually fractionating and being refilled by new primitive liquid. Such a process would imply higher and higher concentrations of hygromagmaphile elements: since these elements are trapped in the liquid, long-life magma chambers would be factories for hygromagmaphile element enrichment.

Finally, we note that the spectrum of hygromagmaphile elements observed in the cores recovered during Leg 65 from a portion of the East Pacific Rise where the opening rate is about $6 \mathrm{~cm} / \mathrm{y}$. is very similar to the spec- trum observed at $22^{\circ} \mathrm{N}$ in the Atlantic Ocean (opening rate: $2 \mathrm{~cm} / \mathrm{y}$.). The idea of the lifetime of a magma chamber as a function of spreading rate is therefore not supported by evidence from trace element geochemistry.

In summary, trace element studies of the basalts recovered on Leg 65 provide the following:

1) Evidence of the removal of olivine, spinel, and(or) clinopyroxene in proportions which varied from unit to unit;

2) No evidence of a clear correlation between the degree of fractionation and either distance from the axis or depth in the crust; and

3) A spectrum of hygromagmaphile elements similar to that observed for the Mid-Atlantic Ridge at $22^{\circ} \mathrm{N}$. Qualitatively, these observations support the existence of discrete magma chambers. Until they have been quantified, however, we would not like to say that large 


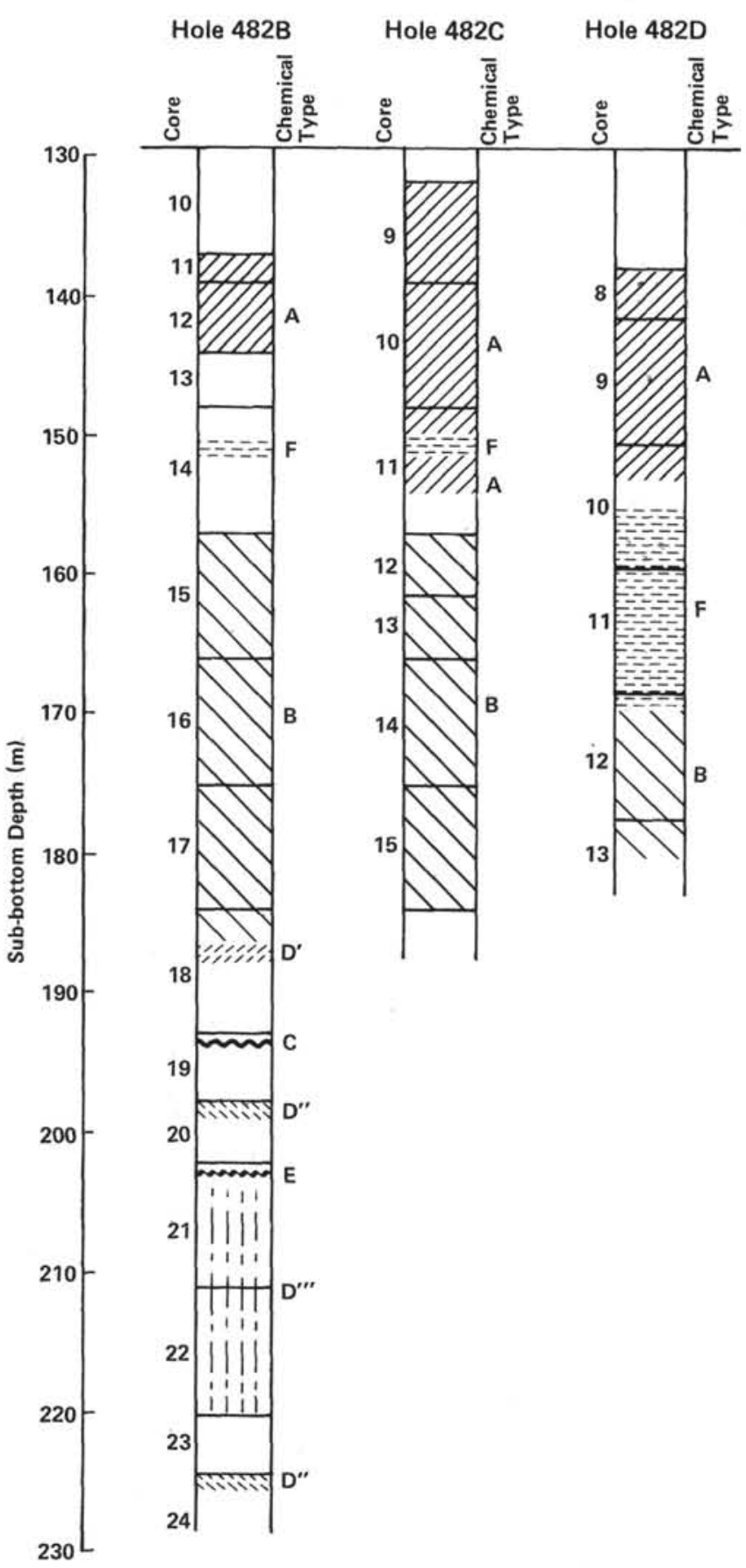

Figure 1. Chemical units vs. depth in Holes 482B, 482C, and 482D.

magma thambers living for a long period of time do not exist. Nevertheless, any model involving large magma chambers will have to account for the apparently incompatible distribution of some of the elements observed in this study and for the field relationships observed between chemical types.

\section{HYGROMAGMAPHILE ELEMENT GEOCHEMISTRY}

On the basis of the classification scheme for the hygromagmaphile elements proposed by Bougault et al. (1979) and their chemical properties, the elements in the

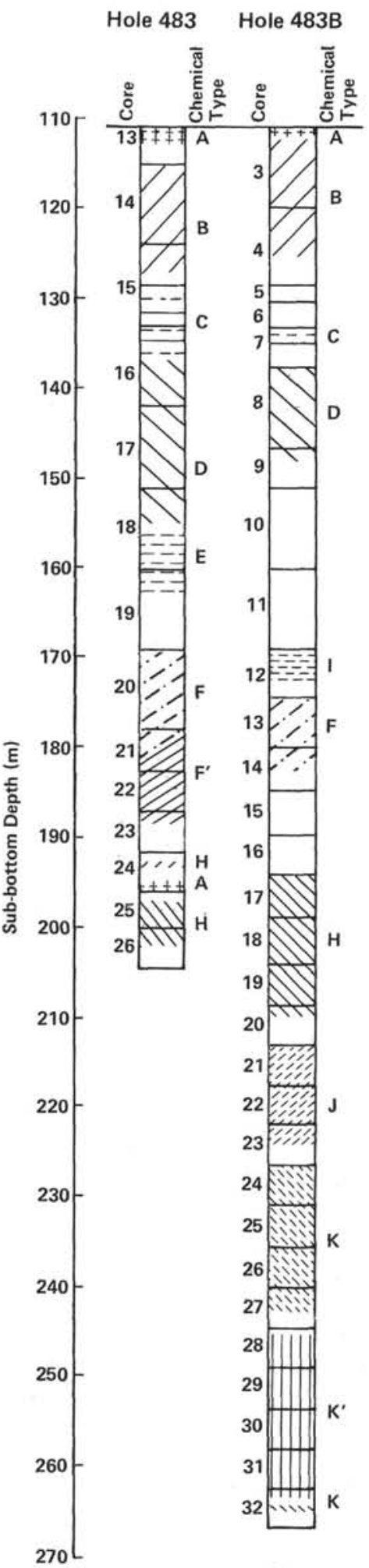

Figure 2. Chemical units vs. depth in Holes 483 and 483B.

pairs $\mathrm{Nb}-\mathrm{Ta}, \mathrm{Zr}-\mathrm{Hf}$, and $\mathrm{Y}-\mathrm{Tb}$ fractionate very little with respect to each other during magma genesis; this property is well known for $\mathrm{Y}$, the position of which coincides with that of $\mathrm{Tb}$ in Coryell-Masuda rare-earth element diagrams. The $\mathrm{Nb} / \mathrm{Ta}, \mathrm{Zr} / \mathrm{Hf}$, and $\mathrm{Y} / \mathrm{Tb}$ ratios in the basalts are close to the ratios observed in "pri- 
mordial chondritic earth" material $(17,40$, and 46 , respectively).

From Tables 4 and 5, in which the average trace element compositions of the chemical types at each site are reported, it is readily seen that all of the $\mathrm{Zr} / \mathrm{Hf}$ ratios lie between 38 and 40 , which confirms that the ratio found previously for oceanic tholeiites is independent of mantle heterogeneity. The $\mathrm{Y} / \mathrm{Tb}$ ratio is close to 50 , which is higher than the presently accepted mean value of 46 for chondrites. We note that this ratio is the same as that which can be deduced from the Leg 54 data (Joron et al., 1980). Nonetheless, before any explanation of this apparent difference is attempted, we must examine for possible sources of analytical error.

A single $\mathrm{Nb}$ determination by XRF techniques is accurate to about $\pm 1 \mathrm{ppm}$ : for this reason, it is not possible to calculate a $\mathrm{Nb} / \mathrm{Ta}$ ratio from a single analysis if the sample has an $\mathrm{Nb}$ concentration of between 1 and 4 $\mathrm{ppm}$. The average $\mathrm{Nb}$ value for each homogeneous unit is known to about $0.5 \mathrm{ppm}$, but this precision is still not sufficient for a $\mathrm{Nb} / \mathrm{Ta}$ calculation. The $\mathrm{Nb} / \mathrm{Ta}$ ratio can be determined statistically, however, from the average values for each type using the least-squares method as indicated in Figure 3. The $\mathrm{Nb} / \mathrm{Ta}$ ratio found by this method is 16.4 , which is very close to the value of $17 \mathrm{ob}-$ served in the Atlantic Ocean, indicating that this ratio is also independent of any mantle heterogeneity.

Except for the small discrepancy observed for the $\mathrm{Y} / \mathrm{Tb}$ ratio (which is probably analytical), the ratios of the pairs of elements which do not fractionate during magma genesis $(\mathrm{Nb} / \mathrm{Ta}, \mathrm{Zr} / \mathrm{Hf}$, and $\mathrm{Y} / \mathrm{Tb})$ are the same for the Atlantic and Pacific Oceans and represent the ratios for a primordial homogeneous Earth of chondritic composition.

Two different $\mathrm{La} / \mathrm{Ta}$ ratios have been found to date: 9, which corresponds to a rare-earth element distribution which is either flat or reflects light rare-earth element enrichment, and 18, which reflects light rare-earth element depletion. In the Atlantic, a ratio of 9 has been found for the FAMOUS area, for $45^{\circ} \mathrm{N}$, and for the Reykjanes Ridge, and a value of 18 has been found on each side of the Mid-Atlantic Ridge at $22^{\circ} \mathrm{N}$ (Legs 45 and 46) and at $25^{\circ} \mathrm{N}$ on $110 \mathrm{~m}$.y. old crust (Legs 51, 52, and 53). In the Pacific Ocean, a value of 9 has been found for rocks from the Emperor Seamount chain (Leg 55, Cambon et al., 1980) and 18 was measured on the East Pacific Rise (Leg 54, Joron et al., 1980). The average values of the $\mathrm{La} / \mathrm{Ta}$ ratio for the chemical types at Sites 482 and 483 are plotted in Figure 4: the value of 20 determined by the least-squares method is close to that associated with the Leg 54 basalts at $9^{\circ} \mathrm{N}$ and indicates light rare-earth element depletion.

Recently, the classification of those elements whose ions have a rare-gas electron structure has been improved by taking theoretical considerations into account (Bougault, 1980) and by incorporating the results of comparative geochemical studies. The hygromagmaphile character of an element not only depends upon its ion size but also upon its ability to form complexes in the liquid (Ringwood, 1955; Treuil, 1973). The hygromagmaphile character, $\Phi$, of an element is described by the relation,

$$
\Phi=\left[a\left(\Delta R_{\mathrm{i}}\right)^{2}+1\right]+\phi,
$$

Table 4. Average trace element composition, $\bar{x}$, of chemical types at Site $482 .^{\text {a }}$

\begin{tabular}{|c|c|c|c|c|c|c|c|c|c|c|c|c|c|c|c|c|c|c|c|c|c|c|c|c|c|}
\hline \multirow{2}{*}{\multicolumn{2}{|c|}{$\begin{array}{c}\text { Chemical } \\
\text { Type }\end{array}$}} & \multirow[b]{2}{*}{$\mathrm{Sc}$} & \multirow[b]{2}{*}{$\mathrm{Ti}$} & \multirow[b]{2}{*}{ V } & \multirow[b]{2}{*}{$\mathrm{Cr}$} & \multirow[b]{2}{*}{$\mathrm{Mn}$} & \multirow[b]{2}{*}{$\mathrm{Fe}$} & \multicolumn{2}{|c|}{$\mathrm{Co}$} & \multicolumn{2}{|c|}{$\mathrm{Ni}$} & \multirow[b]{2}{*}{$\mathrm{Zn}$} & \multirow[b]{2}{*}{$\mathrm{Rb}$} & \multirow[b]{2}{*}{$\mathrm{Sr}$} & \multirow[b]{2}{*}{$\mathrm{Y}$} & \multirow[b]{2}{*}{$\mathrm{Zr}$} & \multirow[b]{2}{*}{$\mathrm{Nb}$} & \multirow[b]{2}{*}{ Cs } & \multirow[b]{2}{*}{$\mathrm{La}$} & \multirow[b]{2}{*}{$\mathrm{Eu}$} & \multirow[b]{2}{*}{$\mathrm{Tb}$} & \multirow[b]{2}{*}{$\mathrm{Hf}$} & \multirow[b]{2}{*}{$\mathrm{Ta}$} & \multirow[b]{2}{*}{ Th } & \multirow[b]{2}{*}{$\mathrm{U}$} \\
\hline & & & & & & & & XRF & $\overline{\text { NAA }}$ & XRF & $\overline{N A A}$ & & & & & & & & & & & & & & \\
\hline \multirow[t]{2}{*}{ A } & $\bar{x}$ & 40 & 10,570 & 351 & 295 & 1,405 & 80,333 & 41.3 & & 72.8 & & 75.9 & $<1$ & 108.6 & 46.6 & 122.6 & 2.4 & - & 3.0 & 1.41 & 0.89 & 3.16 & 0.14 & 0.09 & 0.04 \\
\hline & $a$ & & 155 & 8 & 24 & 156 & 1,925 & 1.4 & & 5.2 & & 5.1 & & 2.7 & 1.3 & 3.0 & 0.5 & & 0.1 & 0.07 & 0.02 & 0.14 & 0.01 & 0.01 & \\
\hline \multirow[t]{2}{*}{ B } & $\bar{x}$ & 39.2 & 7,737 & 309 & 179 & 1,335 & 77,812 & 42 & & 58.7 & & 68.7 & $<1$ & 93.1 & 35.5 & 79.7 & 1.8 & - & 1.94 & 1.04 & 0.68 & 2.09 & 0.09 & 0.05 & \\
\hline & 0 & 1.3 & 113 & 12 & 10 & 73 & 1,291 & 1.4 & & 2.6 & & 4.3 & & 1.1 & 0.8 & 3.0 & 0.6 & & 0.15 & 0.07 & 0.02 & 0.09 & 0.01 & 0.01 & \\
\hline \multirow[t]{2}{*}{ F } & $\bar{x}$ & 38 & 8,400 & 301 & 264 & 1,394 & 77,682 & $43^{1.4}$ & & 95 & & 73.7 & $<1$ & 93.2 & 37.5 & 87.5 & 1.5 & - & 2.1 & 1.15 & 0.74 & 2.3 & 0.1 & 0.07 & \\
\hline & $\sigma$ & & 69 & 6 & 11 & & 367 & & & 6 & & 2.5 & & 2.9 & 0.6 & 4.0 & & & & & & & & & \\
\hline
\end{tabular}

a Values shown in ppm; $\sigma$ represents one standard deviation.

Table 5. Average trace element composition, $\bar{x}$, of chemical types at Site $483 .^{\mathrm{a}}$

\begin{tabular}{|c|c|c|c|c|c|c|c|c|c|c|c|c|c|c|c|c|c|c|c|c|c|c|c|c|c|}
\hline \multirow{2}{*}{\multicolumn{2}{|c|}{$\begin{array}{c}\text { Chemical } \\
\text { Type }\end{array}$}} & \multirow[b]{2}{*}{ Sc } & \multirow[b]{2}{*}{$\mathrm{Ti}$} & \multirow[b]{2}{*}{$\mathrm{v}$} & \multirow[b]{2}{*}{$\mathrm{Cr}$} & \multirow[b]{2}{*}{$\mathrm{Mn}$} & \multirow[b]{2}{*}{$\mathrm{Fe}$} & \multicolumn{2}{|c|}{ Co } & \multicolumn{2}{|c|}{$\mathrm{Ni}$} & \multirow[b]{2}{*}{$\mathrm{Zn}$} & \multirow[b]{2}{*}{$\mathrm{Rb}$} & \multirow[b]{2}{*}{ St } & \multirow[b]{2}{*}{$\mathrm{Y}$} & \multirow[b]{2}{*}{$\mathrm{Zr}$} & \multirow[b]{2}{*}{$\mathrm{Nb}$} & \multirow[b]{2}{*}{ Cs } & \multirow[b]{2}{*}{$\mathrm{La}$} & \multirow[b]{2}{*}{$\mathrm{Eu}$} & \multirow[b]{2}{*}{$\mathrm{Tb}$} & \multirow[b]{2}{*}{ Hf } & \multirow[b]{2}{*}{$\mathrm{Ta}$} & \multirow[b]{2}{*}{ Th } & \multirow[b]{2}{*}{$\mathrm{U}$} \\
\hline & & & & & & & & XRF & NAA & XRF & NAA & & & & & & & & & & & & & & \\
\hline A & $\vec{x}$ & 36 & 7500 & 300 & 175 & 1300 & 76200 & 40 & & 67 & & 70 & $<1$ & 90 & 33 & 70 & 1 & - & 1.7 & 1.1 & 0.66 & 1.97 & 0.08 & 0.05 & \\
\hline \multirow[t]{2}{*}{ B } & $\bar{x}$ & 37 & 7200 & 268 & 375 & 1300 & 72230 & 42 & & 71 & & 60 & $<1$ & 113.5 & 31 & 76.3 & 1.9 & - & 1.7 & 1.04 & 0.6 & 1.84 & 0.1 & 0.4 & \\
\hline & $\sigma$ & & 107 & 9 & 5 & & 1520 & & & 2 & & & & 2 & 0.8 & 1.5 & 0.5 & & & & & & & & \\
\hline \multirow[t]{2}{*}{ C } & $\dot{x}$ & & 8052 & 292 & 244 & 1400 & 77715 & 43.2 & & 58 & & 70 & $<1$ & 95 & 35.4 & 77 & 1.5 & & & & & & & & \\
\hline & $\sigma$ & & 130 & 9 & 8 & & 1700 & 1.3 & & 1.3 & & & & & 0.9 & 2 & & & & & & & & & \\
\hline \multirow[t]{2}{*}{ D } & $\bar{x}$ & & 5750 & 210 & 345 & 1250 & 66300 & 43 & & 109 & & 53 & $<1$ & 97 & 25.4 & 60 & 0.5 & - & & & & & & & \\
\hline & $\sigma$ & & 66 & 7 & 10 & & & & & 4 & & & & 3 & 0.9 & 2 & 0.4 & & & & & & & & \\
\hline \multirow[t]{2}{*}{ F } & $\bar{x}$ & & 11250 & 368 & 205 & 1500 & 84750 & 43 & & 68.4 & & 87 & $<1$ & 103 & 50 & 136 & 2.9 & - & 3.7 & 1.52 & 1.05 & 3.62 & 0.19 & 0.16 & \\
\hline & $\sigma$ & & 256 & 15 & 10 & & & 1.4 & & 3 & & & & 2 & 1 & 3 & 0.8 & & & & & & & & \\
\hline$F^{\prime}$ & $\bar{x}$ & & 9934 & 332 & 279 & 1450 & 80000 & 42 & & 84 & & 80 & $<1$ & 99 & 44.4 & 117 & 2.3 & - & 3.1 & 1.06 & 0.81 & 3.12 & 0.16 & 0.14 & \\
\hline & $\sigma$ & & 300 & 12 & 23 & & & 1 & & 8 & & & & 2 & 1.5 & 7 & 0.5 & & & & & & & & \\
\hline $\mathrm{H}$ & $\bar{x}$ & & 12825 & 405 & 166 & 1500 & 88135 & 44 & & 66 & & 90 & $<1$ & 106 & 55 & 155 & 3.9 & - & 4.4 & 1.5 & 1.12 & 3.96 & 0.22 & 0.19 & \\
\hline & $\sigma$ & & 270 & 18 & 8 & & 2860 & 1.6 & & 5 & & 4 & & 3 & 1 & 3.6 & 0.5 & & & & & & & & \\
\hline $\mathbf{J}$ & $\bar{x}$ & & 9885 & 340 & 219 & 1500 & 80500 & 44 & & 61 & & 79 & $<1$ & 108 & 41 & 107 & 2.3 & - & 3.3 & 0.99 & 0.8 & 2.73 & 0.15 & 0.11 & \\
\hline & $\sigma$ & & 123 & 7 & 4 & & & & & 6 & & & & 1 & 1 & 1 & 0.5 & & & & & & & & \\
\hline K & $\bar{x}$ & & 11150 & 374 & 186 & 1400 & 83000 & 45 & & 64 & & 87 & $<1$ & 114 & 47 & 123 & 2.7 & - & & & & & & & \\
\hline & 0 & & 212 & 16 & 19 & & & 2.5 & & 6 & & 5 & & 5 & 0.8 & 5 & 0.5 & & & & & & & & \\
\hline
\end{tabular}

a Values shown in ppm; $\sigma$ represents one standard deviation. 


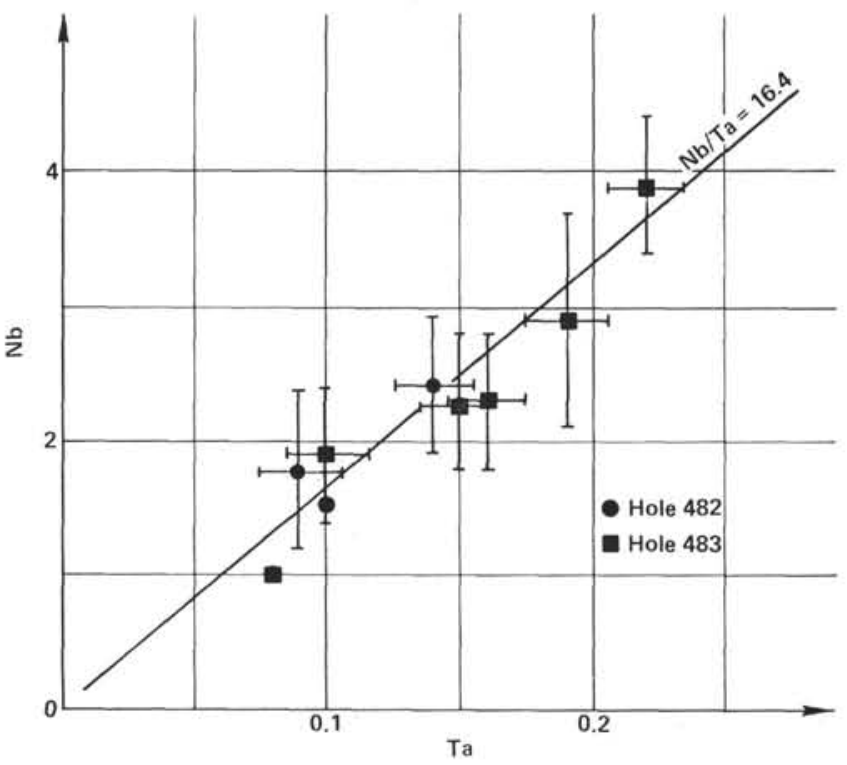

Figure 3. Least-squares determination of $\mathrm{Nb} / \mathrm{Ta}$ ratio for Sites 482 and 483 , using average values for each chemical type.

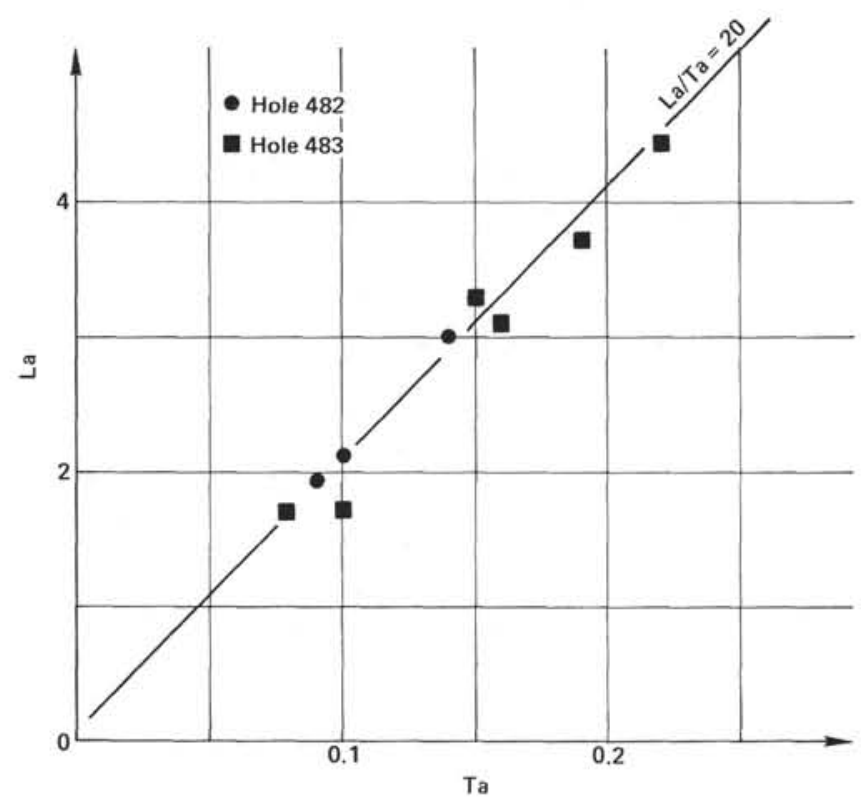

Figure 4. Least-squares determination of $\mathrm{La}$ /Ta ratio for Sites 482 and 483 , using average values for each chemical type.

where $\Delta R_{\mathrm{i}}$ is the difference between the major and trace element ionic radii, $\phi$ is the ionic potential of the trace element, and $a$ is a parameter which defines the relative importance of the two functions, $\Delta R_{\mathrm{i}}$ and $\phi$, which are responsible for the hygromagmaphile character of the element. According to this relation, $\Delta R_{\mathrm{j}}$ is thus a measure of the inability of an element to enter into a crystal structure and $\phi$ is a measure of its ability to form complexes in a magma. This theoretical approach, which is in perfect agreement with the results of comparative geochemical studies, leads to the classification of hygromagmaphile elements indicated on the $x$ axis of Figure 5 . This classification is also in agreement with the classi- fication chosen by Wood (1979). In an extended Coryell-Masuda diagram, which includes both rare-earth elements and nonrare-earth elements, it can be observed not only that $\mathrm{Y}$ has to be plotted very close to $\mathrm{Tb}$, but that $\mathrm{Hf}, \mathrm{Zr}$, and $\mathrm{Ti}$ plot close to $\mathrm{Sm}$ and that $\mathrm{Ta}$ and $\mathrm{Nb}$ plot close to La. It can also be seen that V is the least hygromagmaphile and Th the most hygromagmaphile element. In this diagram, the nonrare-earth elements plot on the same continuous line as the rare-earth elements and with the same precision.

As can be seen in Figure 5, Chemical Types A and B at Site 482 show a typical "light rare-earth" depleted pattern consistent with the $\mathrm{La}$ / Ta ratios discussed above. For these patterns, the normalized concentrations of $\mathrm{Ta}$ and $\mathrm{Nb}$ are lower than the La normalized concentrations by a factor of 2. Sample 482B-19-1, 96-100 cm has the highest absolute concentrations of hygromagmaphile elements; although only XRF data are available, it is evident from the $\mathrm{Nb}$ concentration that this sample also shows the characteristic "light rare-earth" depleted pattern. Chemical Types B, D, H, and J at Site 483 are plotted in Figure 6 . Types $\mathrm{H}$ and $\mathrm{J}$ very probably show a negative Eu anomaly. Type D, for which only XRF data are available, also shows a "light rare-earth" depleted character, its $\mathrm{Nb}$ value being the lowest in Hole 483. The most primitive basalt encountered in Hole 485 (Sample $483-11-3,82-83 \mathrm{~cm}$ ) and another representative sample from the same hole are plotted in Figure 7.

It can be concluded that all of the samples recovered during Leg 65 were derived from typical mantle material depleted in the highly hygromagmaphile elements: their $\mathrm{La} / \mathrm{Ta}$ ratios are close to 18 ; the samples are similar in all respects to the basalts collected during the CYAMEX expedition (1981); and they show the same extended Coryell-Masuda patterns as the Leg 54 samples from $9^{\circ} \mathrm{N}$.

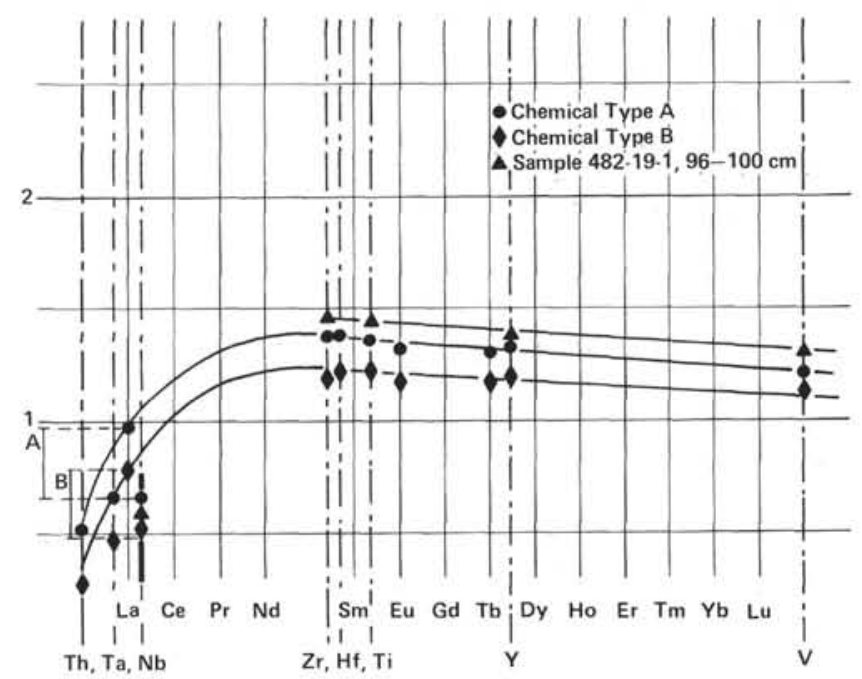

Figure 5. Extended Coryell-Masuda diagram for Chemical Types A, $\mathrm{B}$, and $\mathrm{C}$ at Site 482. (Values shown for A and B represent average concentrations; Type C is represented only by Sample 482-19-1, $96-100 \mathrm{~cm}$, for which only XRF data are available. The ordinate positions for $\mathrm{Nb}-\mathrm{Ta}$ and $\mathrm{La}$ are shown on the left of the figure for Types A and B. The error bar for Nb is shown for Sample 482$19-1,91-100 \mathrm{~cm}$ as a vertical bar.) 


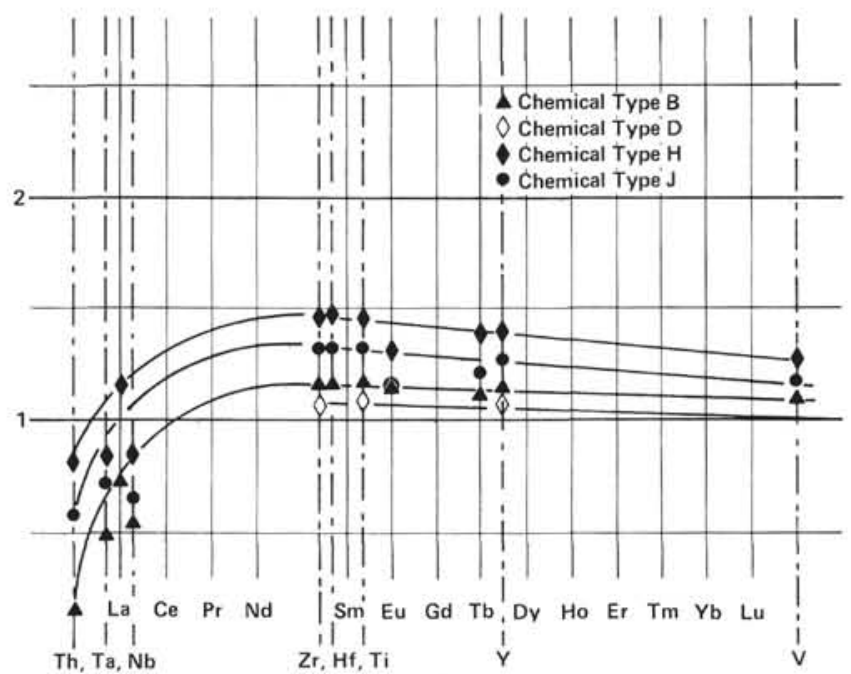

Figure 6. Extended Coryell-Masuda diagram for Chemical Types B, $\mathrm{D}, \mathrm{H}$, and $\mathrm{J}$ at Site 483 . (Values shown represent average concentrations.)

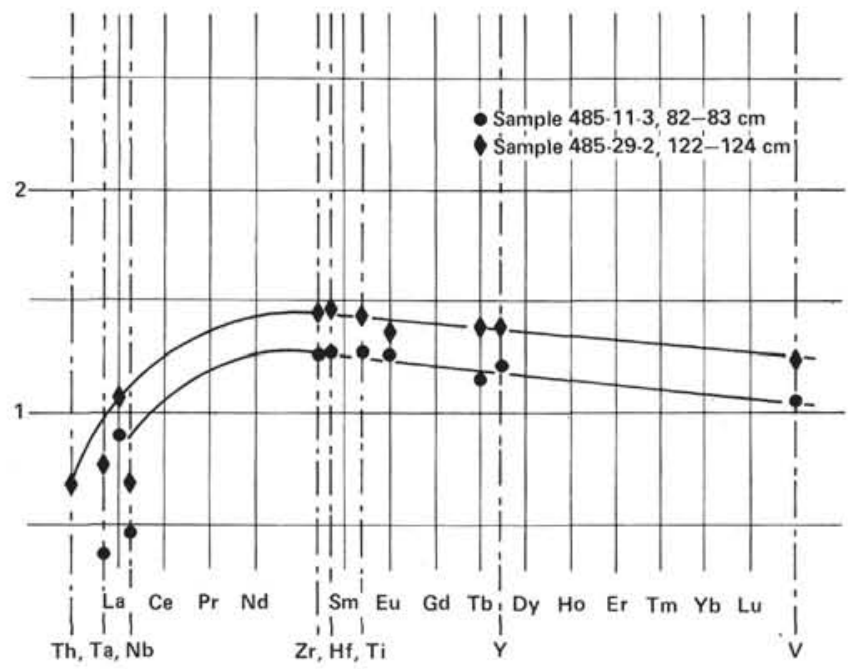

Figure 7. Extended Coryell-Masuda diagram for Samples 485-11-3, $82-83 \mathrm{~cm}$ and $485-29-2,122-124 \mathrm{~cm}$.

These results, when compared with those from the Atlantic, suggest that large regions of the mantle are chemically homogeneous.

\section{CONCLUSIONS}

From the major and trace element data presented above, it has been possible to draw several important conclusions concerning the origin and evolution of basaltic magmas in the mouth of the Gulf of California.

First, the trace element data have refined and confirmed the shipboard classification of the Leg 65 basalts based on major element data. The vertical correlation between the chemical units observed in the various holes drilled at Sites 482 and 483 is remarkable. When considered with the drilling results from earlier legs, it should be possible to put constraints on possible volumes of homogeneous lavas emitted during single volcanic events.
Second, the limited role of fractional crystallization, the range of variation of the hygromagmaphile elements, and the field data at the mouth of the Gulf all appear to be inconsistent with the existence of a large magma chamber under the ridge for any extended period of time.

Finally, the $\mathrm{Nb} / \mathrm{Ta}, \mathrm{Zr} / \mathrm{Hf}$, and $\mathrm{Y} / \mathrm{Tb}$ ratios measured for the Leg 65 basalts show the commonly encountered values of 17,40 , and 50 , respectively, and extended Coryell-Masuda plots of the data show a characteristic light rare-earth depleted pattern and a $\mathrm{La} / \mathrm{Ta}$ ratio close to 18 (as opposed to the ratio 9 observed in material with a flat to light rare-earth enriched pattern). These results are very similar to the results obtained for samples from the East Pacific Rise at $21^{\circ} \mathrm{N}$ and $9^{\circ} \mathrm{N}$ and tend to demonstrate the chemical homogeneity of the mantle in this part of the ocean.

\section{ACKNOWLEDGMENTS}

We thank J. Francheteau and D. Needham for discussing this article and improving the manuscript. This is contribution no. 694 of the Centre Océanologique de Bretagne.

\section{REFERENCES}

Bougault, H., 1977. Major elements: Analytical chemistry on board and preliminary results. In Aumento, F., Melson, W. G., et al., Init. Repts. DSDP, 37: Washington (U.S. Govt. Printing Office), 643-657.

1980. Contributions des éléments de transition à la compréhension de la genèse des basaltes océaniques. Analyse des éléments traces dans les roches par spectromètrie de fluorescence $\mathrm{X}$ [Thèse]. Université Paris VII.

Bougault, H., Cambon, P., Corre, O., et al., 1979. Evidence for variability of magmatic processes and upper mantle heterogeneity in the axial region of the Mid-Atlantic Ridge near $22^{\circ} \mathrm{N}$ and $36^{\circ} \mathrm{N}$. Tectonophysics, 55:11-34.

Bougault, H., Cambon, P., and Toulhouat, H., 1977. X-ray spectrometry analysis of trace elements in rocks-Correction for instrumental interferences. X-Ray Spectrom., 6(2):66-72.

Bougault, H., Joron, J. L., and Treuil, M., 1979. Alteration, fractional crystallization, partial melting, mantle properties from trace elements in basalts recovered in the North Atlantic. Deep Drilling Results in the Atlantic Ocean: Ocean Crust. (Geodynamic Project: Scientific Report 48) Maurice Ewing Series 2:352-367.

Bryan, W. B., and Thompson, G., 1979. Compositional variation in a steady state zoned magma chamber: Mid-Atlantic Ridge at $36^{\circ}$ 50 ' N. Tectonophysics, p. 55.

Cambon, P., Joron, J. L., Bougault, H., et al., 1980. Emperor Seamounts: Trace elements in transitional tholeiites, alkali basalts and Hawaiites: Mantle homogeneity or heterogeneity and magmatic processes. In Jackson, E. D., Koizumi, I., et al., Init. Repts. DSDP, 55: Washington (U.S. Govt. Printing Office), 585-598.

CYAMEX Science Team, 1981. First manned submersible dives on the East Pacific Rise at Lat. $21^{\circ} \mathrm{N}$ (Project Rita): General results. Mar. Geophys. Res., 4:365-379.

Joron, J. L., Briqueu, L., Bougault, H., et al., 1980. East Pacific Rise, Galapagos Spreading Center and Siqueiros Fracture Zone. Hygromagmaphile elements: A comparison with the North Atlantic. In Rosendahl, B. R., Hekinian, R., et al., Init. Repts. DSDP, 54: Washington (U.S. Govt. Printing Office), 725-736.

Melson, W. G., Rabinowitz, P. D., Natland, J. H., et al., 1979. Cruise objectives and major results, analytical procedures and explanatory notes. In Melson, W. G., Rabinowitz, P. D., et al., Init. Repts. DSDP, 45: Washington (U.S. Govt. Printing Office), 5-20.

O'Hara, M. J., 1977. Geochemical evolution during fractional crystallization of a periodically refilled magma chamber. Nature, 266: 503-507.

Rhodes, J. M., Dungan, M. A., Blanchard, D. P., and Long, P. E., 1979. Magma mixing at mid-oceanic ridges: Evidence from basalts drilled near $22^{\circ} \mathrm{N}$ on the Mid-Atlantic Ridge. Tectonophysics, p. 55 . 
Ringwood, A. E., 1955. The principles governing trace element behavior during magmatic crystallization. Part II: The role of complex formation. Geochim. Cosmochim. Acta, 7:242-254.

RISE Project Group, 1980. East Pacific Rise: Hot springs and geophysical experiments. Science, 207(4438):1421-1433.

Treuil, M., 1973. Critères pétrologiques, géochimiques et structuraux de la genèse et de la différenciation des magmas basaltiques: Exemple de l'Afar [Thèse]. Université d'Orléans.
Treuil, M., Jaffrezic, H., Deschamps, N., et al., 1973. Analyse des lauthanides, du hafnium, du scandium, du chrome, du manganèse, du cobalt, du Cuivre et du zinc dans les minéraux et les roches par activation neutronique. J. Radioanal. Chem., 18: $55-68$.

Wood, D. A., 1979. A variably veined suboceanic upper mantle: Genetic significance for mid-ocean ridge basalts from geochemical evidence. Geology, 7:499-503. 